\title{
Statistical Models of Global Langmuir Mixing
}

\author{
Qing $\mathrm{Li}^{\mathrm{a}, \mathrm{b}, *}$, Baylor Fox-Kemper ${ }^{\mathrm{a}, \mathrm{b}}, \varnothing_{\text {yvind Breivik }}^{\mathrm{c}}$, Adrean Webb ${ }^{\mathrm{d}}$ \\ ${ }^{a}$ Dept. of Earth, Environmental and Planetary Sciences, Brown University, Providence, \\ Rhode Island, USA \\ ${ }^{b}$ Institute at Brown for Environment and Society, Brown University, Providence, Rhode \\ Island, USA \\ ${ }^{c}$ Norwegian Meteorological Institute, and Geophysical Institute, University of Bergen, \\ Bergen, Norway \\ ${ }^{d}$ Dept. of Ocean Technology, Policy, and Environment, The University of Tokyo, Kashiwa, \\ Chiba, Japan
}

\begin{abstract}
The effects of Langmuir mixing on the surface ocean mixing may be parameterized by applying an enhancement factor which depends on wave, wind, and ocean state to the turbulent velocity scale in the $K$-Profile Parameterization. Diagnosing the appropriate enhancement factor online in global climate simulations is readily achieved by coupling with a prognostic wave model, but with significant computational and code development expenses. In this paper, two alternatives that do not require a prognostic wave model, (i) a monthly mean enhancement factor climatology, and (ii) an approximation to the enhancement factor based on the empirical wave spectra, are explored and tested in a global climate model. Both appear to reproduce the Langmuir mixing effects as estimated using a prognostic wave model, with nearly identical and substantial improvements in the simulated mixed layer depth and intermediate water ventilation over control simulations, but significantly less computational cost. Simpler approaches, such as ignoring Langmuir mixing altogether or setting a globally constant Langmuir number, are found to be deficient. Thus, the consequences of Stokes depth and misaligned wind and waves are important.

Keywords: Langmuir mixing, Statistical modeling, KPP, Climate model
\end{abstract}

\footnotetext{
${ }^{*}$ Corresponding author.

Email address: qing_li_1@brown.edu (Qing Li)
}

Preprint submitted to Ocean Modelling

March 20, 2017

(C) 2017. This manuscript version is made available under the Elsevier user license http://www.elsevier.com/open-access/userlicense/1.0/ 


\section{Introduction}

The effects of ocean surface waves, particularly the Langmuir turbulence induced by the interaction between the mean flow and the Stokes drift (Craik and Leibovich, 1976; McWilliams et al., 1997; Ardhuin et al., 2008; Teixeira, 2011) or equivalently the Stokes shear force (Suzuki and Fox-Kemper, 2016), are important in enhancing the vertical mixing within the ocean surface boundary layer and potentially contribute to the deepening of the ocean mixed layer ( $\mathrm{Li}$ et al., 1995; Sullivan et al., 2007; Grant and Belcher, 2009; Kukulka et al., 2009; Huang and Qiao, 2010; Cavaleri et al., 2012; Harcourt, 2013; Fan and Griffies, 2014; Li et al., 2016), though some observations suggest that the direct penetration of Langmuir circulation is confined within the upper half of the mixed layer (Weller and Price, 1988; Thorpe et al., 2003). Neglect of the Langmuir turbulence induced mixing, or Langmuir mixing, in most climate models is partially responsible for the persistent shallow mixed layer biases in the Southern Ocean and elsewhere (Belcher et al., 2012; D'Asaro et al., 2014).

Improvements in the simulated mixed layer depth (MLD), deep water ventilation and ocean subsurface temperature are found in Li et al. (2016, hereafter LW16) when Langmuir mixing is parameterized in the National Center for Atmospheric Research (NCAR) Community Earth System Model (CESM) following the scalings found to encapsulate Large Eddy Simulations (Van Roekel et al., 2012). In LW16, a prognostic wave model, WAVEWATCH III (Tolman, 2009), is coupled with CESM to provide the necessary wave information for the Langmuir mixing parameterization. However, running such a prognostic wave model in a climate model is computationally expensive, even with a relatively coarse resolution wave model. With WAVEWATCH III running on a $3.2^{\circ} \times$ $4^{\circ}$ latitude-longitude grid with 25 frequency and 24 directional bins, LW16 report $36 \%$ and $28 \%$ increase in computational cost for ocean-wave only and fully coupled simulations, respectively, in the low-resolution CESM with nominal $3^{\circ}$ resolution ocean model and T31 atmosphere (Shields et al., 2012). Depending on the choice of other component models in the coupled climate system, 
running WAVEWATCH III on the same coarse resolution in CESM with the nominal $1^{\circ}$ resolution ocean model and a higher-resolution atmosphere increases the computational cost by $3-10 \%$ (Table 1). Modern global wave hindcasts and projections typically use $1^{\circ}$ spatial resolution or higher (e.g., Rascle et al., 2008; Rascle and Ardhuin, 2013; Hemer et al., 2013; Breivik et al., 2015), which is at least 50 times more computationally expensive. Therefore, this project explores computationally cheaper possibilities. Statistical modeling of Langmuir mixing effects-with varying degrees of sophistication-is a natural step, along with more efficient numerical approaches (e.g., Webb, 2013).

Statistical descriptions of the ocean surface waves are common practice in both research and operations (Bouws, 1998). Due to their apparently random phases, the statistical description of ocean surface waves begins with a power spectrum of surface height displacements, based on the random-phase/amplitude model (Pierson et al., 1955; Holthuijsen, 2007). Empirical shapes fitted to wave spectra are proposed based on the wave measurements and expected dynamical scalings, e.g., the Phillips spectrum (Phillips, 1958) for the high frequency part of the spectrum, the Pierson-Moskowitz spectrum (Pierson and Moskowitz, 1964) for fully developed wind-waves and the JONSWAP spectrum (Hasselmann et al., 1973) and modified versions (e.g., Donelan et al., 1985) for growing wind waves. The peak frequency is often assumed to be related to the wind speed as empirically estimated from observations (e.g., Bouws, 1998). Other wave parameters can then be derived from the empirical wave spectrum, including the Stokes drift (Kenyon, 1969; McWilliams and Restrepo, 1999; Webb and FoxKemper, 2011; Breivik et al., 2014, 2016), which is of the greatest interest here. These references assume a one-dimensional wave spectrum without directional spreading or crossing swell. These effects can be estimated and accounted for by making some assumptions on the directional spreading distribution and using observations (Donelan et al., 1985; Feddersen, 2004; Webb and Fox-Kemper, 2015).

For the purpose of parameterizing Langmuir mixing, empirical wave spectra are particularly useful. The Stokes drift depends mostly on the high frequency 
part of the wave spectrum, which is reasonably well described under common conditions by the empirical spectra (Webb and Fox-Kemper, 2011, 2015). As shown by Breivik et al. (2016), the Phillips spectrum reasonably approximates the Stokes drift profile, which is a key ingredient of the LES scaling (Harcourt and D'Asaro, 2008; Van Roekel et al., 2012). Since high frequency wind waves are strongly and rapidly coupled with the local wind, predictions to the Stokes drift profiles and thereby the Langmuir mixing effects can be made from only the local wind field using the empirical spectra. Of course, the wind and full wave spectrum, including swell, are normally not in equilibrium, especially in regions away from the storm tracks (Hanley et al., 2010). Swell are usually misaligned with the local wind and may affect Langmuir turbulence (Van Roekel et al., 2012; McWilliams et al., 2014). Nevertheless, the mean effect of the multidirectional waves on the Stokes drift can be estimated empirically (Webb and Fox-Kemper, 2015) and thereby taken into account. In this paper an approximation to the Langmuir mixing effects based on the Phillips spectrum with directional spreading will be assessed.

Another way to statistically represent the effects of the Langmuir mixing is simply replacing the prognostic wave model with a data model, or climatological lookup table, as the source of the wave information. This technique is commonly used to stand in for climate system components in uncoupled climate simulations and model evaluations, e.g., the Coordinated Ocean-ice Reference Experiments (CORE) for global ocean-ice simulations (Griffies et al., 2009). A global wave climatology can be derived from global wave hindcasts forced by global wind reanalyses, such as Rascle et al. (2008); Rascle and Ardhuin (2013). However, as discussed in LW16 and as will be briefly reviewed in Section 2, the effects of Langmuir mixing are represented by an enhancement factor, which is a nonlinear function of the wave parameters (e.g., Stokes drift) as well as other parameters (e.g., surface friction velocity, boundary layer depth, etc.). Deriving enhancement factors from a wave climatology neglects some necessary cross-correlations among the wave parameters (Myrhaug, 2013, 2015). Therefore, a climatology of the enhancement factor itself, which is estimated from 
an ocean-wave coupled simulation, is assessed here. The feasibility of this approach is supported by observational evidence that the surface wave induced enhancement of vertical mixing within the ocean surface boundary layer does not significantly correlate with other parameters on short timescales of days to weeks (D'Asaro, 2001; Tseng and D'Asaro, 2004). Remaining issues in this approach, to be addressed here, are how stable such a climatological estimate is under climate change, what temporal and spatial resolution is required for good performance of the climatology, and whether the feedback from climate variables onto the enhancement factor are significant.

The structure of this paper is as follows. The Langmuir mixing parameterization scheme from LW16 is briefly reviewed in Section 2. In Sections 3 and 4, two options to approximate the Langmuir mixing effects which are based on (i) an enhancement factor climatology and (ii) empirical wave spectra are explored as computationally cheaper substitutes for the prognostic wave model in LW16. The results from implementing these two options in CESM are presented in Section 5 , followed by a discussion in Section 6 . This paper ends with the main conclusions in Section 7. Note that the statistical modeling approaches explored in this paper aim to represent only Langmuir mixing effects. Whether they work or not in this regard does not imply applicability for other ocean surface wave effects.

\section{Langmuir mixing parameterization and basic simulation}

Classical scaling suggests that the bulk vertical turbulent kinetic energy (VKE) in the ocean surface boundary layer (OSBL), $\left\langle w^{2}\right\rangle$, scales with the squared friction velocity, $u^{* 2} \equiv|\boldsymbol{\tau}| / \rho_{0}$, where $\boldsymbol{\tau}$ is the surface wind stress and $\rho_{0}$ is the surface ocean density. The ratio of these two, $\left\langle w^{2}\right\rangle / u^{* 2}$, is suggested to be about 0.6 , based mostly on studies of the atmospheric boundary layer and laboratory experiments. However, observation (D'Asaro, 2001; Tseng and D'Asaro, 2004; D'Asaro et al., 2014) and large eddy simulation (LES) based studies (Skyllingstad and Denbo, 1995; McWilliams et al., 1997; Harcourt and 
D’Asaro, 2008; Grant and Belcher, 2009; Van Roekel et al., 2012), show that for the OSBL in the presence of surface waves, this ratio can be significantly greater than the canonical value, due to additional energy extracted from surface waves. This behavior was also confirmed by the rapid distortion theory (RDT) calculations of Teixeira (2011). As the real OSBL is likely to be in the regime of parameter space where the Langmuir turbulence competes with both convection- and shear-driven turbulence in order to energize vertical turbulent mixing (Li et al., 2005; Belcher et al., 2012), an OSBL model without surface waves, such as the $K$-Profile Parameterization (KPP, Large et al., 1994), will likely underestimate vertical mixing within the OSBL.

McWilliams and Sullivan (2000) suggest that the extra mixing induced by the Langmuir turbulence can be parameterized by applying an enhancement factor, $\mathcal{E}$, to the turbulent velocity scale used in KPP,

$$
W=\frac{k u^{*}}{\phi} \mathcal{E}
$$

with $k=0.4$ the von Kármán constant and $\phi$ the dimensionless flux profile. Here $W$ is the velocity scale from which the vertical eddy diffusivity in KPP is defined. It essentially represents the root mean square value of the vertical turbulent velocity, and retains its dependence on winds and convection through $u^{*}$ and the convective velocity scale $w^{*}$ (see more detail in Large et al., 1994). McWilliams and Sullivan (2000) propose a formula for $\mathcal{E}$,

$$
\mathcal{E}=\sqrt{1+0.08 L a_{\mathrm{t}}^{-4}}
$$

to fit a few LES results, where $L a_{\mathrm{t}}=\sqrt{u^{*} / u_{0}^{\mathrm{S}}}$ is the turbulent Langmuir number (McWilliams et al., 1997) and $u_{0}^{\mathrm{S}}=\left|\boldsymbol{u}^{\mathrm{S}}(0)\right|$ the magnitude of the surface Stokes drift velocity. By incorporating WAVEWATCH III into CESM, LW16 find that (2), as well as a modified formula by Smyth et al. (2002), generally introduce too much mixing in the extratropical regions in CESM, though sensitivity differs in other models (Fan and Griffies, 2014).

Based on the LES studies by Harcourt and D'Asaro (2008) and Van Roekel 
et al. (2012), LW16 propose a formula,

$$
\mathcal{E}=|\cos \alpha| \sqrt{1+\left(1.5 L a_{\mathrm{SL}, \mathrm{proj}}\right)^{-2}+\left(5.4 L a_{\mathrm{SL}, \mathrm{proj}}\right)^{-4}} .
$$

The surface layer averaged and projected Langmuir number,

$$
L a_{\mathrm{SL}, \text { proj }}=\sqrt{\frac{u^{*} \cos (\alpha)}{\left|\left\langle\boldsymbol{u}^{\mathrm{S}}\right\rangle_{\mathrm{SL}}\right| \cos \left(\theta_{\mathrm{ww}}-\alpha\right)}},
$$

is used to account for both the effects of the Stokes depth and the wind-wave misalignment. Here, $\theta_{\mathrm{ww}}$ is the angle between wind and waves, and $\left\langle\boldsymbol{u}^{\mathrm{S}}\right\rangle_{\mathrm{SL}}$ the surface layer averaged Stokes drift,

$$
\left\langle\boldsymbol{u}^{\mathrm{S}}\right\rangle_{\mathrm{SL}}=\frac{1}{H_{\mathrm{SL}}} \int_{-H_{\mathrm{SL}}}^{0} \boldsymbol{u}^{\mathrm{S}}(z) d z,
$$

with $\boldsymbol{u}^{\mathrm{S}}(z)$ the Stokes drift profile and $H_{\mathrm{SL}}$ the surface layer depth, taken here as proportional to the critical Richardson number boundary layer depth calculated by KPP: $H_{\mathrm{SL}}=0.2 H_{\mathrm{BL}}$. The angle between wind and Langmuir cells $\alpha$ is estimated based on the law of the wall (Van Roekel et al., 2012),

$$
\alpha \approx \tan ^{-1}\left[\frac{\sin \left(\theta_{\mathrm{ww}}\right)}{\frac{u^{*}}{u_{0}^{\mathrm{s}} k} \ln \left(\left|H_{\mathrm{BL}} / z_{1}\right|\right)+\cos \left(\theta_{\mathrm{ww}}\right)}\right],
$$

with $z_{1}$ the onset depth of the law of the wall, which is taken as four times the significant wave height (Thorpe, 2007).

Note that the enhancement factor applied to the turbulent velocity scale is the only modification to KPP here. The particular coefficient choices in (3) are trained against the LES results with and without waves (Van Roekel et al., 2012; Harcourt and D'Asaro, 2008). No retuning of coefficients is allowed to improve the climate model after values are set to match the LES results. Notable alternative approaches, such as paying more attention to the wavefree state by retuning the parameters in KPP to reproduce the LES results without wave forcing (Reichl et al., 2016) and changing the KPP boundary layer depth in LW16 to simulate the wave-driven entrainment, can be used readily in the framework developed here. However, a more comprehensive set of LES is required to justify these approaches and the examination of these approaches 
is beyond the scope of the present work, which focuses solely on changing the source of wave information without changes to parameterization settings.

Results from two sets of simulations will be discussed in this paper: the Ocean-Wave only simulations and the Atmosphere-Ocean-Land-Ice-Wave fully coupled simulations. Both consist of a control simulation without Langmuir mixing parameterization (CTRL) and simulations with Langmuir mixing enhancement factor from various sources: a prognostic wave model, WAVEWATCH III (WW3), the Data Wave climatology (DWAV) and the Theory Wave estimate (TWAV). All simulations are summarized in Table 2 and will be detailed in the following three sections.

In the Ocean-Wave only simulations, the ocean model was running on a nominal $1^{\circ}$ resolution grid under CORE-II inter-annual forcing (62 years forcing cycle). A suffix, FULL, is used for the simulation with the Data Wave climatology to distinguish it from the four sensitivity test simulations detailed in Section 5.2. All the simulations with the Langmuir mixing parameterization were branched from the control simulation at the beginning of the model year 195 (corresponding to the forcing year 1956) and run throughout a fourth CORE-II inter-annual forcing cycle to the model year 248 (forcing year 2009). The data from the last 50 years of the simulation (corresponding to forcing year 1960 to 2009 ) is analyzed here.

The configurations of the fully coupled simulations are the same as described in LW16, except with higher resolution. Here a roughly $2^{\circ}$ grid is used for the atmosphere and the land model, whereas a nominal $1^{\circ}$ grid is used for the sea ice and the ocean model. Simulations with the pre-industrial forcing scenario and the 20th century transient scenario are prefixed by PI and 20C, respectively. In the former scenario, the greenhouse gas concentrations were held fixed at the pre-industrial level, and simulations with Langmuir mixing parameterization were branched from the control simulation at the beginning of the model year 21 and run for 80 years. Whereas in the latter scenario, the greenhouse gas concentrations history since 1850 were applied. For both scenarios the data from the last 30 years of the simulations are analyzed. 


\section{The Data Wave Model}

\subsection{Enhancement factor statistics}

As the enhancement factor, $\mathcal{E}$, is the only wave parameter in our simple Langmuir mixing parameterization, a deeper look at its statistics is warranted. The essential hypothesis to be tested here is that a data wave model, i.e., a climatology of $\mathcal{E}$, can reproduce the Langmuir mixing effects introduced by the computationally expensive prognostic wave model.

A practical data wave model for Langmuir mixing requires that (i) the ocean model should not be too sensitive to high-frequency variations of $\mathcal{E}$, or to covariances of $\mathcal{E}$ and other variables $\left(u^{*}, \mathrm{MLD}\right.$, etc.) that occur on short timescales, and (ii) the low-frequency $\mathcal{E}$ climatology should be robust under climate change (i.e., changes in forcing such as wind and temperature). Of course, high-frequency and climate-induced changes to $u^{*}$, temperature, etc., are $e x$ pected, but the data wave model will pass this variability along unhindered-it relies only on stability of the enhancement factor. Since monthly mean statistics are typically examined in climate models as they average over synoptic weather, a month is taken as a reasonable timescale dividing the high- and low-frequency. Conveniently, a month also lies between the zero-crossing and integral timescales of the Stokes drift autocorrelation ${ }^{1}$ (Haney et al., 2015).

The first requirement may result from either an extremely fast linear response or an extremely slow response that is not necessarily linear. By design, $\mathcal{E}$ applied to the turbulent velocity scale in KPP instantaneously changes the boundary layer depth. Tests varying the sampling frequency of $\mathcal{E}$ from three hours to a month show that the monthly mean ocean model response is not sensitive to high frequency variations of $\mathcal{E}$. On the other hand, observations show no significant correlation between the amount of surface wave induced enhancement of vertical mixing and other wind or wave properties on timescales of days

\footnotetext{
${ }^{1}$ The zero-crossing timescale is defined as the time lag at which the autocorrelation function first makes a zero-crossing. The integral timescale is defined as $\tau=\int_{0}^{\infty} c(t) \mathrm{d} t$, where $c(t)$ is the autocorrelation function with lag $t$ (Tennekes and Lumley, 1972).
} 
to weeks (D'Asaro, 2001; Tseng and D'Asaro, 2004). One may speculate that this is a result of the relatively slow evolution of major shifts in the boundary layer depth or seasonal regime and the fact that faster changes needed are well approximated by changes to $u^{*}$ or convective forcing without the need to also vary the enhancement factor.

The second requirement is tested by analyzing the low frequency statistics of $\mathcal{E}$ from an ocean-wave coupled simulation. This simulation, denoted as WW3 hereafter, was forced by the inter-annually varying Coordinated Ocean-ice Reference Experiments phase II atmospheric datasets (CORE-II, Large and Yeager, 2009). The coupled system is virtually the same as described in LW16, and the Langmuir mixing parameterization scheme corresponds to the case VR12-MA therein, as outlined in Section 2. The only difference is that here $\mathcal{E}$ is calculated in the wave model, and is the only wave variable passed on to the ocean model. This code modification does not significantly influence results. However, under this code algorithm a data wave model is easily implemented, as the statistics of $\mathcal{E}$ simply replace the prognostic wave model $\mathcal{E}$. The nonlinear relations in (3) - (6) make it impossible to reconstruct the $\mathcal{E}$ climatology from separate climatologies of $L a_{\mathrm{t}}, \alpha, H_{B L}$ and the spectral significant wave height, $H_{\mathrm{m}_{0}}$, because these variables are significantly related (Myrhaug, 2013, 2015).

To evaluate the robustness under changes in the mean wind forcing, the $\mathcal{E}$ statistics from the last 10 years of a prognostic wave model simulation (corresponding to forcing year 2000-2009, denoted as period $P_{2}$ ) are compared with that from the first 10 years (corresponding forcing year 1960-1969, denoted as period $P_{1}$ ). Table 3 shows the changes in the $10-\mathrm{m}$ wind, the friction velocity, the significant wave height, the surface Stokes drift magnitude and the enhancement factor, respectively, in different regions. The differences between these two periods are measured by two methods, the normalized mean difference, $\mathrm{NMD}=\frac{\overline{x\left(P_{2}\right)}-\overline{x\left(P_{1}\right)}}{\overline{x\left(P_{1}\right)}} \times 100 \%$, and the normalized root mean square difference, $\mathrm{NRMSD}=\sqrt{\overline{\left(\frac{x\left(P_{2}\right)-x\left(P_{1}\right)}{x\left(P_{1}\right)}\right)^{2}}} \times 100 \%$, in which $\overline{()}$ represents the spatial and temporal average over 10 years and $x$ represents any of the wave variables. As 
seen in Table 3, during the 50-year analysis window, the global 10-year mean wind increases by $2.5 \%$, correspondingly, significant wave height $3.9 \%$, Stokes drift $3.8 \%$. However, the mean enhancement factor is virtually unchanged. This difference among wave statistics in response to the changing wind forcing is particularly significant for the Southern Hemisphere extra-tropical regions (consistent with Hemer et al., 2013). The normalized root mean square difference shows a similar lack of sensitivity in enhancement factor when compared to the other wave and boundary layer statistics. The enhancement factor is more stable than other wave statistics under changes in the mean wind forcing and presumably also under projected climate change (e.g., Hemer et al., 2013; Fan et al., 2014). This result is partially expected because $\mathcal{E}$ is a dimensionless number describing a relation between the Langmuir mixing and the wind forcing, rather than a wave state that depends directly on the forcing, although it is certainly possible for dimensionless numbers to change as much as, or more than, their constituents. While the enhancement factor is found on the fly from a combination of more variable physical parameters, such as $u^{*}$, apparently the majority of the variability in wave forcing is captured without variation in the enhancement factor. To the extent that such a climatology is based purely on basin geometry, large-scale wind patterns (e.g., storm tracks), and seasonality, it should be robust in the face of climate change-indeed it is only imperceptibly changed over 50 years of intensive late 20th century climate change. While important covariances in wave parameters make $\mathcal{E}$ stable, $\mathcal{E}$ itself has no important covariances with the other variables. Therefore, a data model for the Langmuir mixing effects is possible.

\subsection{An enhancement factor climatology}

A monthly $\mathcal{E}$ climatology is created from the simulation WW3, by taking the monthly average of the 6-hourly output of $\mathcal{E}$ over the last 10 years of the simulation (2000-2009). One could take the monthly average over a different 10-year time window or make the average window longer, but the differences in the resulting $\mathcal{E}$ climatology are trivial. Grid points with sea ice fraction greater 
than 0.05 for longer than half the average window are eliminated from the average. Consistently, the same ice fraction threshold is used when applying the enhancement factor in the ocean model to make sure the Langmuir mixing effects only being applied to ice free regions. Note that this sea ice threshold is much smaller than that used in WAVEWATCH III $(0.67$, with another smaller value of 0.33 for adding ice to obstructions). One may infer from this large range that there is considerable uncertainty about the effects of Langmuir turbulence near the sea ice margin. Physically, surface waves are attenuated in the marginal ice zone due to viscous damping and multiple wave scattering by floe edges (Squire and Moore, 1980; Bennetts and Squire, 2012), and a wave-ice interaction model is required to represent these effects (See, e.g., Williams et al., 2013).

This monthly $\mathcal{E}$ climatology is illustrated in Fig. 1 and is termed the Data Wave model hereafter. The most striking feature of the Data Wave model is that it has a strong seasonal cycle, which will be shown to have non-negligible effects. Its value is relatively big in local summer and small in local winter. At first, this pattern is counterintuitive, as storms are most vigorous in local winter and so are the waves. However, as will be detailed in Section $6, \mathcal{E}$ measures the relative effects of waves to other forcing such as wind and convective forcing. In winter convection dominates and waves are not expected to have as large relative effects as in summer (as observations show in Belcher et al., 2012). This behavior is built into the surface layer averaged Stokes drift (4). Another important feature is that $\mathcal{E}$ is relatively small as compared with previous studies (e.g. Fan and Griffies, 2014), in which a fully developed sea and aligned wind and waves are assumed. For comparison, using the typical value of $L a_{\mathrm{t}}=0.3$ which results from empirical scalings for fully-developed waves (Pierson and Moskowitz, 1964) and assuming wind and wave alignment, (2) gives $\mathcal{E}=3.30$ while the equivalent from Van Roekel et al. (2012) gives $\mathcal{E}=1.52$. The values in Fig. 1 rarely exceed $\mathcal{E}=1.5$. As shown in LW16 these assumptions cause too much mixing.

A comparison between the Data Wave and the original 10 years of prognostic model data from the 6-hourly WW3 data is made in the density-shaded scatter 
plots of $\mathcal{E}, u_{0}^{\mathrm{S}}$ and the magnitude of the surface layer averaged Stokes drift $u_{\mathrm{SL}}^{\mathrm{S}}=\left|\left\langle\boldsymbol{u}^{\mathrm{S}}\right\rangle_{\mathrm{SL}}\right|$ in Figs. 2a, 2b and 2c. The yellow, green and blue color represent the highest $0-30 \%, 31-60 \%$ and $61-90 \%$ centered distribution ${ }^{2}$, respectively. The monthly mean data in the Data Wave climatology is linearly interpolated back to a 6-hourly frequency to match sample size with the WW3 data. As expected, the monthly mean removes the extreme values and therefore all the three variables in the Data Wave climatology have a narrower distribution than the WW3 data. Despite the spread of the scatter, the distributions for $\mathcal{E}$ and $u_{0}^{\mathrm{S}}$ are centered on the one-to-one reference line, indicating no significant bias is introduced to these variables by the monthly mean. However, the Data Wave overestimates $u_{\mathrm{SL}}^{\mathrm{S}}$, presumably due to the fact that the intermittent storm-induced swells affect $u_{\mathrm{SL}}^{\mathrm{S}}$ more than $u_{0}^{\mathrm{S}}$.

The Data Wave model only approximates $\mathcal{E}$ within the limits of the simulation quality of WW3. Practically, the detailed spatial distribution of $\mathcal{E}$ could be different from that shown in Fig. 1 if the simulated large scale wind and wave patterns were different, for example, in the case of fully coupled simulations with a prognostic atmosphere model, where wind is biased, or in the case of using a better wave model, where the wave physics is improved so that the simulated Stokes drift is different given the same wind forcing. However, those deviations are from the model biases in the simulated wind and waves. This is the main reason of deriving the $\mathcal{E}$ climatology from the ocean-wave only simulations (in which the wind forcing is based on observations) rather than fully coupled simulations. Potential improvement on the $\mathcal{E}$ climatology could probably be achieved by coupling the ocean model to a wave model with higher resolution and better wave physics, which is beyond the scope of this paper. An estimate of potential improvement from a high-resolution, data assimilating wave model will be presented in experiment DWAV-RA13 below.

${ }^{2}$ Note that the number of scatters within the yellow, green and blue areas are equal $(30 \%$ of total number of scatters), but the density is highest in the yellow area. 


\section{The Theory Wave Model}

While the Data Wave model mimics the mean effects from a prognostic wave model, another approach towards a statistical modeling of the Langmuir mixing effects is to approximate $\mathcal{E}$ from wave theory. The essential question this section addresses is: can we reasonably approximate $\mathcal{E}$ from only the atmospheric and oceanic parameters that are available in the climate model? From (3) - (6), the ingredients to approximate $\mathcal{E}$ are the Stokes drift profile, the boundary layer depth and the surface friction velocity. As the latter two are readily available from the ocean model, only a reasonable approximation to the Stokes drift profile remains. For simplicity Stokes drift from swell will not be explicitly included in the derivation, though the bulk effect is roughly accounted for by a magnitude loss coefficient (see more discussion in Section 4.3 and 4.4). Swell is remotely generated and thus affected by a complex history of wind forcing and wave propagation. Therefore, their accurate representation requires a prognostic wave model.

\subsection{Approximating the Stokes drift profile}

The basis of approximating the Stokes drift profile from an empirical wave spectrum is that the Stokes drift scales with the third moment ${ }^{3}$ of a depthdependent wave spectrum, which accentuates the higher frequency part of the spectrum near the surface (Webb and Fox-Kemper, 2011). Breivik et al. (2014, 2016) show that a reasonable approximation to the full Stokes drift profile could be made by using the Phillips spectrum (Phillips, 1958) with a simple peak cutoff for low frequencies, based on the assumption that the Phillips spectrum approximates the largest contribution to Stokes drift near the surface, the high frequency part of the real spectrum, reasonably well. With the Phillips spec-

${ }^{3}$ The $n$th moment of the wave spectrum is defined as $m_{n}=\int_{0}^{\infty} \int_{-\pi}^{\pi} \omega^{n} S(\omega, \theta) d \theta d \omega$. 
trum,

$$
S_{\text {Phil }}= \begin{cases}\alpha g^{2} \omega^{-5}, & \omega>\omega_{\mathrm{p}} \\ 0, & \omega \leq \omega_{\mathrm{p}}\end{cases}
$$

in which $\alpha=0.0083$ is the Phillips' parameter, the Stokes drift profile is (Breivik et al., 2014, 2016)

$$
\begin{aligned}
\boldsymbol{u}_{\text {Phil }}^{\mathrm{S}}(z) & =\hat{\boldsymbol{e}}^{\mathrm{w}} \frac{2}{g} \int_{0}^{\infty} \omega^{3} S_{\text {Phil }}(\omega) \mathrm{e}^{2 k z} \mathrm{~d} \omega \\
& =\hat{\boldsymbol{e}}^{\mathrm{w}} u_{0, \text { Phil }}^{\mathrm{S}}\left[T_{1}\left(k_{\mathrm{p}}, z\right)-T_{2}\left(k_{\mathrm{p}}, z\right)\right],
\end{aligned}
$$

where

$$
\begin{aligned}
& T_{1}(k, z)=\mathrm{e}^{2 k z}, \\
& T_{2}(k, z)=\sqrt{2 \pi k|z|} \operatorname{erfc}(\sqrt{2 k|z|}) .
\end{aligned}
$$

Here $\hat{\boldsymbol{e}}^{\mathrm{w}}$ is the (unidirectional) wave direction, for now assumed to be constant with depth, $u_{0, \text { Phil }}^{\mathrm{S}}=\left|\boldsymbol{u}_{\text {Phil }}^{\mathrm{S}}(0)\right|=2 \alpha \mathrm{g} / \omega_{\mathrm{p}}$ the surface Stokes drift, $\operatorname{erfc}(\cdot)$ the complementary error function, $k_{\mathrm{p}}=\omega_{\mathrm{p}}^{2} / g$ the peak wavenumber and $z \leq 0$ the depth. The deep-water wave dispersion relation $\omega^{2}=g k$ is used.

While the unidirectional wave approximation is used here with the Phillips spectrum, as most other empirical wave spectra (Webb and Fox-Kemper, 2011), Webb and Fox-Kemper (2015) show that the unidirectional wave approximation tends to overestimate the magnitude of Stokes drift as wave spreading and multidirectional waves tend to prevent part of the wave energy from contributing to the Stokes drift. No swell is accounted for in the Phillips spectrum, and discussion of these effects is postponed until Section 4.3. Directional spreading of wind waves, however, can be approximated following Webb and Fox-Kemper (2015) who employed a directional-Stokes-drift-component derived from the spectral spreading function by Donelan et al. (1985), hereafter DHH,

$$
\boldsymbol{u}_{\text {Phil-s }}^{\mathrm{S}}(z)=\hat{\boldsymbol{e}}^{\mathrm{w}} \frac{2}{g} \int_{0}^{\infty} H_{\mathrm{DHH}}\left(\omega / \omega_{\mathrm{p}}\right) \omega^{3} S_{\text {Phil }}(\omega) \mathrm{e}^{2 k z} \mathrm{~d} \omega,
$$


where

$$
H_{\mathrm{DHH}}\left(\omega / \omega_{\mathrm{p}}\right)= \begin{cases}\frac{0.52-3.3\left(\omega / \omega_{\mathrm{p}}\right)+8.9\left(\omega / \omega_{\mathrm{p}}\right)^{2}}{1-3.4\left(\omega / \omega_{\mathrm{p}}\right)+8.9\left(\omega / \omega_{\mathrm{p}}\right)^{2}}, & 0.56<\omega / \omega_{\mathrm{p}} \leq 0.95, \\ \frac{0.98-0.19\left(\omega / \omega_{\mathrm{p}}\right)+0.0058\left(\omega / \omega_{\mathrm{p}}\right)^{2}}{1-0.26\left(\omega / \omega_{\mathrm{p}}\right)+0.12\left(\omega / \omega_{\mathrm{p}}\right)^{2}}, & 0.95<\omega / \omega_{\mathrm{p}}<1.6, \\ 0.777, & \text { otherwise, }\end{cases}
$$

390

is the Padé-approximated, DHH directional-Stokes-drift-component. Here the mean wave direction is chosen to be $\hat{\boldsymbol{e}}^{\mathrm{w}}$ (which is constant with depth when only directional spreading is considered). Substituting (7) and (12) into (11) (details in Appendices A and B.1),

$$
\begin{aligned}
\boldsymbol{u}_{\text {Phil-s }}^{\mathrm{S}}(z) \approx \hat{\boldsymbol{e}}^{\mathrm{w}} & u_{0, \text { Phil-s }}^{\mathrm{S}} \\
& \times \begin{cases}1, & z=0, \\
1.26\left[T_{1}\left(k_{\mathrm{p}}, z\right)-B_{1}(z) T_{2}\left(k_{\mathrm{p}}, z\right)\right] & \\
-0.188\left[T_{1}\left(k_{\mathrm{p}}^{*}, z\right)-B_{2}(z) T_{2}\left(k_{\mathrm{p}}^{*}, z\right)\right], & z<0\end{cases}
\end{aligned}
$$

where

$$
\begin{aligned}
& B_{1}(z)=1+\frac{0.0469}{2 k_{\mathrm{p}}|z|}, \\
& B_{2}(z)=1+\frac{0.503}{2 k_{\mathrm{p}}^{*}|z|} .
\end{aligned}
$$

Here $k_{\mathrm{p}}^{*}=1.6^{2} k_{\mathrm{p}}=2.56 k_{\mathrm{p}}$ and $u_{0, \text { Phil-s }}^{\mathrm{S}}=\left|\boldsymbol{u}_{\text {Phil-s }}^{\mathrm{S}}(0)\right| \approx 0.811 u_{0, \text { Phil }}^{\mathrm{S}}$ is the surface Stokes drift including spreading effects. The effect of spreading is to reduce the surface Stokes drift by nearly $20 \%$.

The Stokes drift averaged over a layer from $z=z_{0}\left(z_{0}<0\right)$ to $z=0$ is 
(details in Appendix B.2),

$$
\begin{aligned}
\left\langle\boldsymbol{u}_{\text {Phil-s }}^{\mathrm{S}}\right\rangle_{z_{0}} \approx & \hat{\boldsymbol{e}}^{\mathrm{w}} u_{0, \text { Phil-s }}^{\mathrm{S}}\{0.715 \\
& +\left(\frac{0.151}{k_{\mathrm{p}}\left|z_{0}\right|}-0.840\right)\left[1-T_{1}\left(k_{\mathrm{p}}, z_{0}\right)\right] \\
& -\left(0.840+\frac{0.0591}{k_{\mathrm{p}}\left|z_{0}\right|}\right) T_{2}\left(k_{\mathrm{p}}, z_{0}\right) \\
& +\left(\frac{0.0632}{k_{\mathrm{p}}^{*}\left|z_{0}\right|}+0.125\right)\left[1-T_{1}\left(k_{\mathrm{p}}^{*}, z_{0}\right)\right] \\
& \left.+\left(0.125+\frac{0.0946}{k_{\mathrm{p}}^{*}\left|z_{0}\right|}\right) T_{2}\left(k_{\mathrm{p}}^{*}, z_{0}\right)\right\}
\end{aligned}
$$

This formula is needed to evaluate the Theory Wave $L a_{\text {SL,proj }}$ for an arbitrary boundary layer depth using (4).

\subsection{Estimating the peak wavenumber}

In (16) the only unknowns are the peak wavenumber, $k_{\mathrm{p}}$, and the surface magnitude of the Stokes drift, $u_{0, \text { Phil-s. }}^{\mathrm{S}}$. The peak wavenumber could be estimated from the Pierson-Moskowitz spectrum (Pierson and Moskowitz, 1964; Bouws, 1998). But a more direct constraint for our purposes determines the peak wavenumber and other parameters by requiring that the Stokes drift profile yields a given Stokes transport and a given surface Stokes drift. Following Breivik et al. (2016), a general peak wavenumber could be estimated from the surface Stokes drift and the total Stokes transport,

$$
k_{\text {Phil }}=\frac{u_{0, \text { Phil }}^{\mathrm{S}}}{2 V^{\mathrm{S}}}\left(1-\frac{2 \beta}{3}\right) \approx 0.167 \frac{u_{0, \text { Phil }}^{\mathrm{S}}}{V^{\mathrm{S}}} \approx 0.206 \frac{u_{0, \text { Phil-s }}^{\mathrm{S}}}{V^{\mathrm{S}}},
$$

where $V^{\mathrm{S}}=\left|\boldsymbol{V}^{\mathrm{S}}\right|$, and $\boldsymbol{V}^{\mathrm{S}}=\int_{-\infty}^{0} \boldsymbol{u}^{\mathrm{S}}(z) d z$. Breivik et al. (2016) find that $\beta=1$ is an adequate choice, as assumed here.

Again, no directional spreading effect is accounted for in (17). An improved formula for the general peak wavenumber with directional spreading effects may be derived by integrating (13), instead of (8), from $z=-\infty$ to $z=0$ to find (details in Appendix B.3)

$$
k_{\text {Phil-s }} \approx 0.176 \frac{u_{0, \text { Phil-s }}^{\mathrm{S}}}{V^{\mathrm{S}}} .
$$


Including directional spreading here is a modest effect, as seen by comparing (18) to (17).

\subsection{Stokes transport and surface Stokes drift}

To close the Theory Wave model, approximations are needed to determine the Stokes transport and the surface Stokes drift from variables available in a climate model. The depth-integrated Stokes drift from the Pierson-Moskowitz spectrum can be used to infer Stokes transport from wind speed, as previously used and assessed for the climate model applications (McWilliams and Restrepo, 1999; Webb and Fox-Kemper, 2011). The resulting Stokes transport scales with the first moment of the wave spectrum, or equivalently the mean frequency and significant wave height (e.g. Breivik et al., 2014, 2015),

$$
V^{\mathrm{S}} \approx 2 \pi c_{1} m_{1}=\frac{2 \pi c_{1}}{16} \bar{f} H_{\mathrm{m}_{0}}^{2} .
$$

Here, $c_{1}$ is a magnitude loss coefficent accounting for directional spreading further discussed below. For the Pierson-Moskowitz spectrum the mean frequency is related to the peak frequency (Webb and Fox-Kemper, 2011) by

$$
\bar{f}=\frac{m_{1}}{m_{0}} \approx 1.296 f_{\mathrm{p}}
$$

The peak frequency and the significant wave height from Pierson-Moskowitz spectrum (Bouws, 1998) are, respectively,

$$
f_{\mathrm{p}} \approx 0.877 \frac{g}{2 \pi U_{19.5}} \approx 0.816 \frac{g}{2 \pi U_{10}},
$$

and

$$
H_{\mathrm{m}_{0}}=4 \sqrt{m_{0}} \approx 0.0246 U_{10}^{2}
$$

${ }_{434}$ Here $U_{19.5}$ is the wind speed at $19.5 \mathrm{~m}$ above the sea surface in $\mathrm{m} \mathrm{s}^{-1}$ and $H_{\mathrm{m}_{0}}$ in $\mathrm{m}$. A generally accepted empirical relation, $U_{19.5} \approx 1.075 U_{10}$ (Holthuijsen, 2007), is used in (21).

While the dominant scaling relationships have been laid out, (19) also includes a magnitude loss coefficient $c_{1}<1$ to account for a number of (presumed 
constant) factors that make the unidirectional estimate $2 \pi m_{1}$ an overestimation for the Stokes transport. Some important factors are: (i) ignoring the directional spreading effects overestimates the Stokes transport by about 17\% (Ardhuin et al., 2009; Breivik et al., 2014; Webb and Fox-Kemper, 2015); (ii) ignoring the effects from swell by the use of empirical spectrum overestimates the Stokes transport (Webb and Fox-Kemper (2015) report a combined effect of directional spreading and multidirectional waves corresponding to $c_{1}=0.698$ ); (iii) using the mean frequency and significant wave height from Pierson-Moskowitz spectrum, which represents fully developed wind-waves, overestimates $m_{1}$ for real ocean surface waves. By evaluating this estimate as compared to the 3-hourly Stokes transport data over a year from a global wave hindcast simulation with WAVEWATCH III (Rascle and Ardhuin, 2013, denoted as RA13 hereafter), $c_{1}=0.667$ reasonably accounts for the combined effects mentioned and appears to correct the overestimation, as illustrated in Fig. 3a. Furthermore, a crude approximation of swell on the Stokes drift versus the Phillips wind-wave spectrum is accounted for in tuning the magnitude loss coefficient $c_{1}$ against RA13. Improvement here is certainly possible, but the goal is to evaluate how well a rough estimate of Langmuir mixing performs.

Finally, an approximation for surface Stokes drift is needed. For simplicity it is assumed to be proportional to the 10-meter wind speed,

$$
u_{0, \mathrm{Wind}}^{\mathrm{S}} \approx c_{2} U_{10}
$$

A proportionality constant of $c_{2}=0.016$ is found to agree with the surface Stokes drift from RA13, as illustrated in Fig. 3b. Note that only the slope in Fig. $3 \mathrm{~b}$ depends on $c_{2}$, and the offset cannot be corrected by the simple approach used here. The systematic overestimation by (23) might be due to contributions from swell, which often reduce the surface Stokes drift (Webb and Fox-Kemper, 2015). 
4.4. Predicting the enhancement factor from $U_{10}, u^{*}$ and $H_{B L}$

With $z_{0}=-0.2 H_{\mathrm{BL}}$ being the depth of surface layer in (16), and $\alpha=\theta_{\mathrm{ww}}=$ 0 in (3) and (4), we now have a formula for $\mathcal{E}$ as a function of the 10-meter wind, $U_{10}$, the surface friction velocity, $u^{*}$, and the boundary layer depth, $H_{\mathrm{BL}}$,

$$
\mathcal{E}_{\text {Theory }}=\mathcal{F}\left(U_{10}, u^{*}, H_{\mathrm{BL}}\right) \text {. }
$$

Normally, $U_{10}, u^{*}$ and $H_{\mathrm{BL}}$ are readily available from any ocean model. Therefore, (24) offers a solution for parameterizing the effects of Langmuir mixing without coupling with a prognostic wave model. This approximation to $\mathcal{E}$ is termed the Theory Wave model hereafter.

Note that both the directional spreading of wind-waves and the remotely generated swell lead to the misalignment of wind and waves, reducing the mean Stokes drift magnitude by $14 \sim 20 \%$ and $7 \sim 23 \%$, respectively (Webb and FoxKemper, 2015). Both effects are approximated in the Theory Wave model. The adoption of $\alpha=\theta_{\mathrm{ww}}=0$ here should be distinguished from assuming aligned wind and waves such as in (26) introduced in the next section. The systematic overestimation of the enhancement factor by assuming $\alpha=\theta_{\mathrm{ww}}=0$ is compensated by the adoption the magnitude loss coefficient, $c_{1}$, in (19).

For quick reference and easy implementation, all the essential equations re- 
quired in the Theory Wave model, (24), are summarized below.

$$
\begin{aligned}
& u_{0}^{\mathrm{S}} \approx 0.016 U_{10}, \\
& V^{\mathrm{S}} \approx 2.67 \times 10^{-5} g U_{10}^{3}, \\
& k_{\mathrm{p}} \approx 0.176 \frac{u_{0}^{\mathrm{S}}}{V^{\mathrm{S}}}, \\
& k_{\mathrm{p}}^{*}=2.56 k_{\mathrm{p}}, \\
& H_{\mathrm{SL}}=H_{\mathrm{BL}} / 5 \text {, } \\
& T_{1}(k, z)=\mathrm{e}^{2 k z}, \\
& T_{2}(k, z)=\sqrt{2 \pi k|z|} \operatorname{erfc}(\sqrt{2 k|z|}), \\
& u_{\mathrm{SL}}^{\mathrm{S}} \approx u_{0}^{\mathrm{S}}\{0.715 \\
& +\left(\frac{0.151}{k_{\mathrm{p}} H_{\mathrm{SL}}}-0.840\right)\left[1-T_{1}\left(k_{\mathrm{p}}, H_{\mathrm{SL}}\right)\right] \\
& -\left(0.840+\frac{0.0591}{k_{\mathrm{p}} H_{\mathrm{SL}}}\right) T_{2}\left(k_{\mathrm{p}}, H_{\mathrm{SL}}\right) \\
& +\left(\frac{0.0632}{k_{\mathrm{p}}^{*} H_{\mathrm{SL}}}+0.125\right)\left[1-T_{1}\left(k_{\mathrm{p}}^{*}, H_{\mathrm{SL}}\right)\right] \\
& \left.+\left(0.125+\frac{0.0946}{k_{\mathrm{p}}^{*} H_{\mathrm{SL}}}\right) T_{2}\left(k_{\mathrm{p}}^{*}, H_{\mathrm{SL}}\right)\right\}, \\
& L a_{\mathrm{SL}}=\sqrt{\frac{u^{*}}{u_{\mathrm{SL}}^{\mathrm{S}}}}, \\
& \mathcal{E}=\sqrt{1+\left(1.5 L a_{\mathrm{SL}}\right)^{-2}+\left(5.4 L a_{\mathrm{SL}}\right)^{-4}} \text {. }
\end{aligned}
$$

All the coefficients are dimensionless except that in the approximation of the Stokes transport, $V^{\mathrm{S}}$, which has units of $\mathrm{m}^{-2} \mathrm{~s}^{-4}$.

A comparison between the Theory Wave model and the WW3 simulation is shown by density-shaded scatter plots of $\mathcal{E}, u_{0}^{\mathrm{S}}$ and $u_{\mathrm{SL}}^{\mathrm{S}}$ in Fig. 2d, 2e and $2 \mathrm{f}$. Each corresponding variable from the Theory Wave model are calculated from (25) with the 6-hourly WW3 data of $U_{10}, u^{*}$ and $H_{\mathrm{BL}}$. The $\mathcal{E}$ distribution of the Theory Wave model resembles the WW3 data, with consistent magnitude and variability. A prime discrepancy is that the Theory Wave model does not allow $\mathcal{E}<1$ whereas WW3 does. This discrepancy is negligible overall (see next 
section). Similar to the comparison with RA13 in Fig. $3 \mathrm{~b}, u_{0}^{\mathrm{S}}$ follows WW3 data except for an offset leading to systematic overestimation, likely from swell contributions (Fig. 2e). The Theory Wave prediction for $u_{\mathrm{SL}}^{\mathrm{S}}$ also agrees with the WW3 data (Fig. 2f), especially for large values. The underestimation of $u_{\mathrm{SL}}^{\mathrm{S}}$ at small values has relatively little impact on the resulting enhancement factor.

Fig. 4 shows the comparison of the 10-year averaged enhancement factor map in January and July between the Theory Wave model and the Data Wave model. Generally the Theory Wave estimates match the spatial variability and the seasonality of the enhancement factor in the Data Wave climatology, except for modest overestimation. Beyond comparing just the climatological mean, the enhancement factor from the Theory Wave estimate has a broader distribution than that from the Data Wave climatology (See Fig. 2a,d).

\section{Data Wave climatology and Theory Wave estimates in CESM}

\subsection{Comparing with WAVEWATCH III}

Both the Data Wave climatology and the Theory Wave estimates are implemented in the Community Ocean Vertical Mixing Project (CVMix, https://github.com/CVMix) and tested in CESM. Appendix C details the CVMix implementation. Two ocean-wave simulations forced by the inter-annually varying CORE-II datasets were performed, denoted as DWAV-FULL and TWAV. These two simulations are identical to WW3 except that WAVEWATCH III is replaced by the Data Wave climatology and the Theory Wave estimates, respectively, as the source of the enhancement factor. All simulations are summarized in Table 2.

Fig. 5 and 6 show the summer and winter mean MLD in both hemispheres in observations, CTRL, WW3, DWAV-FULL and TWAV. The observations are from de Boyer Montégut et al. (2004), updated to include Argo data up through 2012. As in LW16, the definition of mixed layer follows the density threshold method of de Boyer Montégut et al. (2004), locating the depth where the potential density (referenced to surface) changes by $0.03 \mathrm{~kg} \mathrm{~m}^{-3}$ from its surface value. This definition allows direct comparison of the model results 
with observations. Note that Fig. 5b, $5 \mathrm{c}$ and $6 \mathrm{~b}, 6 \mathrm{c}$ correspond to Fig. $3 \mathrm{c}, 3 \mathrm{~g}$ and Fig. 4c, 4g in LW16, respectively, except that here the ocean model uses a finer nominal $1^{\circ}$ grid and the color scale is slightly modified. The results here agree with LW16, suggesting that resolution does not significantly affect the parameterizations of Langmuir mixing, at least while holding the wave model resolution fixed.

Fig. 5d, 5e and 6d, 6e show the results for the case DWAV-FULL and TWAV. They are difficult to distinguish from WW3. Thus, the Data Wave climatology and the Theory Wave estimate retain the Langmuir mixing effects on mean MLD from coupling with WAVEWATCH III.

As a direct comparison with Fig. 6 in LW16, Fig. 7 shows the zonal mean partial pressure of chlorofluorocarbon-11 (pCFC-11) over the Southern Hemisphere. Chlorofluorocarbons (CFC) are commonly used as passive tracers in the ocean model as a measure of the integrated effects of the air-sea interaction and mixing in the ocean (e.g., Fox-Kemper et al., 2011; Danabasoglu et al., 2012). The CFC concentrations in the atmosphere were set (realistically) near the end of the third CORE-II cycle in CTRL (at model year 170) and their concentration history is applied thereafter to the end of the fourth CORE-II cycle in all the simulations. This forcing allows the simulation results to be compared directly against observations from the Global Ocean Data Analysis Project (GLODAP, Key et al., 2004). As the CFC climatology in GLODAP is based on data collected during the World Ocean Circulation Experiment (WOCE) program from 1990 to 1998, the simulated pCFC-11 is averaged over the model year 233, corresponding to the forcing year 1994, providing a central estimate of the observation window for comparison.

Consistent with LW16, including the Langmuir mixing parameterization appears to reduce the low concentration biases of pCFC-11 in the Southern Ocean to a modest extent. Most important here is the agreement in Langmuir mixing effects on pCFC-11 among the WW3, DWAV-FULL and TWAV.

Therefore, it appears that the Data Wave climatology and the Theory Wave estimate perform comparably in terms of Langmuir mixing effects to the prog- 
nostic wave model WAVEWATCH III. In the following section, it will be demonstrated that the consistency among these results is not random, and indeed it is easy to generate other estimates that disagree more.

\subsection{Significance assessment versus contrasting simulations}

Four additional ocean-wave simulations were performed to assess the sensitivity of the ocean model to variations in enhancement factor, denoted as DWAV-CON1, DWAV-CON2, DWAV-AL and DWAV-RA13. To avoid the possible unphysical effects near the sea ice margin, the same sea ice mask from DWAV-FULL was applied to these four simulations. Simulation details are described below; Table 2 summarizes them.

Two simulations with constant enhancement factor at all locations at times $(\mathcal{E}=1.52$ and $\mathcal{E}=1.33)$ were performed, denoted as DWAV-CON1 and DWAVCON2, respectively. The former value results from assuming fully-developed wind wave conditions with $L a_{\mathrm{t}}=0.3$ and ignoring the effects of Stokes depth and misaligned wind and waves. The latter value is the global and time mean of the Data Wave climatology. Thus, comparing this simulation to the Data Wave simulation quantifies the importance of spatial variability and seasonality in the Data Wave climatology. The $\mathcal{E}=1.33$ value also agrees with an enhancement factor of $\sqrt{1.75} \approx 1.32$ to $\sqrt{2} \approx 1.41$ as implied from the buoyant float measurements of mean square vertical velocity by D'Asaro (2001); Tseng and D'Asaro (2004), whereas the former $(\mathcal{E}=1.52)$ value is larger than these observational estimates. The simulation DWAV-CON1 is contrasted against TWAV to emphasize the effect of Stokes depth, and perhaps also misaligned wind and waves to a lesser extent (as TWAV includes some spreading effects). And DWAV-CON2 compared to DWAV-FULL reveals the importance of spatial and seasonal variations. In addition, DWAV-CON1 and DWAV-CON2 together offer an assessment of the ocean model sensitivity to the magnitude of the enhancement factor.

In contrast to all of the other simulations here, the monthly $\mathcal{E}$ climatology in DWAV-RA13 is derived from the dataset of a higher resolution WAVEWATCH 
III global wave hindcast with the latest source term parameterization (Rascle and Ardhuin, 2013). However, as this is not an ocean-wave coupled simulation and there is no boundary layer depth information in this dataset, the enhancement factor is estimated from surface values only, using a different formula optimized to neglect misalignment and Stokes depth effects (Van Roekel et al., 2012), which is

$$
\mathcal{E}_{a}=\sqrt{1+\left(3.1 L a_{\mathrm{t}}\right)^{-2}+\left(5.4 L a_{\mathrm{t}}\right)^{-4}}
$$

To evaluate the importance of high-resolution, another simulation denoted DWAVAL uses this same formula but with the coarse resolution wave model used in WW3 and DWAV-FULL. This simulation corresponds to the VR12-AL parameterization scheme in LW16-neglecting wave-wind misalignment and Stokes depth effects. The greatest difference between DWAV-AL and DWAV-FULL simulations is that the former mixes too strongly in the winter hemisphere extratropical regions. Comparing the DWAV-RA13 and DWAV-AL simulations assesses the possibility for potential improvements in estimating the $\mathcal{E}$ climatology by using output data from wave simulations with higher resolution and probably better wave physics. Note that DWAV-RA13 makes use of better wave data, but of a poor Langmuir mixing parameterization scheme.

To be consistent with the Data Wave climatology the 10-year hindcast RA13 data from 2000 to 2009 was used. The calculation is performed on the original grid $\left(0.5^{\circ}\right.$ spatial resolution) and averaged in time to get the monthly climatology. Then the monthly climatology is interpolated to the coarse resolution wave grid $\left(3.2^{\circ} \times 4^{\circ}\right.$ spatial resolution $)$ as used in our coupled system. As the averaged field is spatially smooth, the information loss by interpolating to the coarse resolution is limited.

The left panels of Fig. 8 illustrate the seasonal cycle of mean MLD biases as compared with observations in the Northern Hemisphere extratropical regions $\left(30^{\circ} \mathrm{N}-90^{\circ} \mathrm{N}\right)$, the equatorial regions $\left(30^{\circ} \mathrm{S}-30^{\circ} \mathrm{N}\right)$ and the Southern Hemisphere extratropical regions $\left(90^{\circ} \mathrm{S}-30^{\circ} \mathrm{S}\right)$, respectively. The right panels of Fig. 8 are the corresponding reductions of root mean square error (RMSE) 
as compared with CTRL. The black curves in the left panels represent the mean biases of the CTRL in which no Langmuir mixing effect is included. Note the persistent shallow biases in the Southern Hemisphere extratropical regions (Fig. 8e). The red, blue and purple curves represent results for WW3, DWAVFULL and TWAV, and the solid, dashed, dotted and dashed-dotted curves in gray are for the simulations DWAV-CON1, DWAV-CON2, DWAV-AL and DWAV-RA13, respectively. Note that adding the Langmuir mixing effects is a major change to modeled behavior, but distinctions among different Langmuir treatments are comparatively small. However, two groups of curves distinguish themselves, color versus gray in Fig. 8.

The consistency among WW3, DWAV-FULL and TWAV (colored curves) is remarkable when compared with other cases (gray curves). In particular, although the mean MLD biases in the Northern Hemisphere winter are increased in all cases as compared with CTRL, the three colored cases are clearly less biased (Fig. 8a). And more importantly, the reductions in RMSE are only found in the three colored cases (Fig. 8b). In the equatorial regions, the three colored cases introduce the least degradation, though DWAV-CON2 and DWAV-AL seems to be also indistinguishable from the colored cases (Fig. 8c, 8d). In the Southern Hemisphere extratropical regions, where improvements are found in all cases, the distinctions between the colored cases and the gray cases are clear in both the mean biases (Fig. 8e) and the RMSE reductions (Fig. 8f).

In all the regions throughout the year, DWAV-CON1 performs the worst. This is expected as an enhancement factor of $\mathcal{E}=1.52$ overestimates the Langmuir mixing effects in most cases, especially in winter. However, this does not imply that $L a_{\mathrm{t}}=0.3$ everywhere is a bad approximation. In fact, $L a_{\mathrm{t}}=0.3$ is a reasonable estimate for most regions (See, e.g., Belcher et al., 2012). Note that in the Theory Wave, $c_{2}=0.016$ in (23) gives $L a_{\mathrm{t}} \approx 0.3$. It is ignoring the Stokes depth effects versus the boundary layer depth by scaling the VKE only with $L a_{\mathrm{t}}$, as well as misalignment, that contributes to the overestimation of the Langmuir mixing effects.

Simply tuning the constant enhancement factor to a smaller and more rea- 
sonable value, e.g., $\mathcal{E}=1.33$ in DWAV-CON2, appears to give a fair approximation to the Langmuir mixing effects, except during winter (summer) in the Northern (Southern) Hemisphere extratropical regions, when $\mathcal{E}=1.33$ appears to be an overestimation (underestimation). Comparing DWAV-CON2 with DWAV-CON1, an increase of the enhancement factor from $\mathcal{E}=1.33$ to $\mathcal{E}=1.52$ roughly deepens the winter mean MLD in the extratropical regions by $15 \mathrm{~m}$ (Fig. 8a and 8e).

The relatively poor performance in DWAV-AL confirms the importance of the Stokes depth and misaligned wind and waves effects. The results are slightly better in DWAV-RA13 (presumably due to better simulated surface Stokes drift) suggesting potential improvements from utilizing a higher resolution prognostic wave model with better wave physics. However, DWAV-RA13 still performs significantly worse than DWAV-FULL, especially in the extratropical regions in winter, which suggests that the improvements from a better wave model are limited by the Langmuir mixing parameterization. Consistently, TWAV includes only the barest essentials of wave prediction physics, but includes the effects of Stokes depth and limited wind-wave misalignment, and delivers a closer approximation to DWAV-FULL and WW3 than DWAV-RA13.

The vertical profiles of the pCFC-11 RMSE reductions as compared with CTRL in different regions are shown in Fig. 9, in which a negative value corresponds to an improvement as compared with CTRL, whereas a positive value corresponds to a degradation. The color coding is the same as Fig. 8. The black dashed curve (CTRL*) in each subfigure represents the case in which the root mean square difference from the GLODAP observation (essentially representing the year 1994) is calculated with the simulated mean pCFC-11 from one year later (corresponding to the year 1995). Since the simulated ventilation is insufficient, as suggested by the low pCFC-11 concentration biases (Fig. 7), allowing the ocean to mix one year longer apparently reduces the root mean square difference from observations. This offers an estimate of the significance of the pCFC-11 RMSE reductions induced by parameterizing Langmuir mixing.

Generally, a greater reduction in the low pCFC-11 concentration biases at 
intermediate depth is associated with a stronger degradation near the surface, all brought by a stronger winter mixing (e.g., comparing DWAV-CON1 with DWAV-CON2, or comparing DWAV-AL with DWAV-FULL). Clearly enhancing the vertical mixing within the ocean surface boundary layer alone cannot fix this problem, but fixes are left to future studies. The important results here are, again, that cases WW3, DWAV-FULL and TWAV (colored curves) agree with each other, and differentiate themselves from the other cases.

\subsection{Climate impacts in fully coupled simulations}

In this section, results from a set of the Atmosphere-Ocean-Land-Ice-Wave fully coupled simulations will be discussed, aiming at assessing the performance of the Data Wave climatology and the Theory Wave estimates in the face of the climate feedbacks. Possible feedbacks are as follows. Langmuir mixing deepens the MLD. As a result, the sea surface temperature (SST) and the subsurface temperature adjust accordingly. In the low- to mid-latitudes, SST drops but the subsurface temperature increases, associated with an increase in the surface heat flux into the ocean. Presumably, the surface wind will adjust to the changed surface conditions such as the atmospheric stability and feeds back to the waves, and thereby the Langmuir mixing.

Note that the impact of changing wind on the Langmuir mixing is twofold. On one hand, a change in wind modifies the enhancement factor by changing the mixing regimes with a different turbulent Langmuir number (e.g., Belcher et al., 2012) or changing the state of wind-wave equilibrium with a different wave age (e.g., Harcourt and D'Asaro, 2008). On the other hand, a change in wind also affects the net effects of Langmuir mixing even with a fixed enhancement factor by changing $u^{*}$ in the turbulent velocity scale in KPP. A fixed enhancement factor corresponds to a fixed wind-wave equilibrium state, e.g., fully developed wind-waves described by Pierson and Moskowitz (1964). Both effects exist in the fully coupled simulation with a prognostic wave model, while only the latter one exists in the Data Wave climatological representation. In the case with Theory Wave estimates the former effect is partially represented, but not as in 
a prognostic wave model, where the wind can modify the enhancement factor through (24). However, since the enhancement factor is robust under change in wind climate, as shown in Section 3.1, it is not expected that the former effect is significant. In fact, as will be shown in this section, the coupled model results with both the Data Wave climatology and the Theory Wave estimate resemble those with WAVEWATCH III-that is, coupled model feedbacks onto enhancment factor are not important.

Both the Data Wave climatology and the Theory Wave estimates were tested under the pre-industrial forcing scenario. To begin this suite of simulations, a control simulation without any Langmuir mixing parameterization was integrated for 100 years (PI-CTRL). Starting at year 21, three simulations including Langmuir mixing effects were branched from PI-CTRL and run for 80 years, one with a prognostic wave model, WAVEWATCH III, denoted as PI-WW3, the second one with the Data Wave climatology, denoted as PI-DWAV, and the third one with the Theory Wave estimate, denoted as PI-TWAV. In addition, the control simulation without any Langmuir mixing parameterization (20CCTRL) and a simulation with WAVEWATCH III (20C-WW3) were performed under the 20th century transient scenario for direct comparison to observations. See Table 2 for a brief summary.

Consistent with the ocean-wave only simulations, the deepening in simulated MLD and enhancement in the vertical mixing of the pCFC11 are found in fully coupled simulations. The results of both PI-DWAV and PI-TWAV resemble that of PI-WW3, thus detailed comparison of these results is not needed. Instead, a focus on climate variables that are indirectly affected by the Langmuir mixing and related feedbacks follows.

Fig. 10 illustrates the vertical profiles of the annual mean ocean temperature error averaged over different regions for both PI (solid lines) and 20C (dashed lines) simulations. The errors are computed as model minus observation. The observations are from the Polar Science Center Hydrographic Climatology (PHC3.0) dataset, which is a combination of the National Oceanographic Data Center's (NODC) World Ocean Atlas (WOA) 1998 (Levitus et al., 1998) 
and an updated version by Steele et al. (2001) for the Arctic Ocean.

Corresponding to the deepening in simulated MLD, enhanced vertical mixing at the sea surface efficiently transports heat downward and mixes up the relatively cooler water from below. As a result, the SST decreases and the subsurface temperature increases. As shown in Fig. 10, the global averaged annual mean SST is cooled by $0.12^{\circ} \mathrm{C}$ in PI-DWAV and $0.13^{\circ} \mathrm{C}$ in PI-TWAV, versus $0.15^{\circ} \mathrm{C}$ in PI-WW3. Whereas the subsurface temperature is warmed by as much as about $0.5^{\circ} \mathrm{C}$ between about $100 \mathrm{~m}$ and $200 \mathrm{~m}$. The cooling in the SST is mostly found in the Southern Ocean, where the deepening of the MLD is most significant. The warming in the subsurface layer is mainly in the equatorial regions. As most of the subsurface layer water in the equatorial regions and the southern hemisphere extratropical regions are biased warm near the surface and biased cool in the subsurface layer, the redistribution of the heat over the upper ocean by the Langmuir mixing partly alleviates the biases in the temperature distribution. The bias reduction is similar among DWAV, TWAV, and WW3 coupled simulations.

Since the boundary layer is deepened by Langmuir mixing effects, the amplitude of the SST seasonal cycle is reduced due to increased heat capacity. This is clearly seen in Fig. 11, which shows the seasonal cycle of the SST averaged over the extratropical regions. Note that in all cases the annual mean is subtracted from the monthly mean to show the phase and amplitude of the seasonal cycle. In both hemispheres the mean SST seasonal cycle amplitude is reduced by about $0.4^{\circ} \mathrm{C}$. This reduces the too strong bias of SST seasonal cycle in the Southern Hemisphere extratropical region by about $35 \%$. However, it does not improve the simulated SST seasonal cycle in the Northern Hemisphere extratropical region significantly, where the dominating bias is the too early warming occurring in the summer.

Consistent with LW16, the salinity does not appear to be significantly affected by the parameterized Langmuir mixing. The sea surface water is only slightly fresher in the Southern Ocean and tropics, and saltier in the subtropical regions. 
All these changes are robust between PI and 20C simulations and are reasonably retained when switching from coupling with a prognostic wave model to the Data Wave climatoloty or the Theory Wave estimation. Therefore, both nonprognostic-model approaches appear to work reasonably well in representing the Langmuir mixing effects in fully coupled simulations.

\section{Discussion}

The Data Wave climatology for the enhancement factor was aimed at mimicking the mean Langmuir mixing effects due to coupling with a prognostic wave model and is based on the relation between the wind forcing and the resulting vertical mixing in the ocean surface boundary layer. Note that a monthly mean enhancement factor does not imply that the high frequency forcing from the wind is smoothed out. It is only a particular realization of the rather stochastic wave state (the enhancement factor from a prognostic wave model) that is replaced with the most likely realization under similar wind forcing (the Data Wave). The high frequency wind forcing still affects the ocean surface vertical mixing through its proportionality with $u^{*}$. The monthly climatology is used here simply to retain the seasonality of the Langmuir mixing effects, which is associated with the seasonality of the forcing regimes as will be discussed below. In fact, the Stokes drift has a relatively persistent character, with the autocorrelation zero crossing time varying from 9 to 15 days for the surface values and longer for the subsurface values (Haney et al., 2015). Therefore, further refining the enhancement factor climatology with intermediate values (e.g., daily) does not seem to be necessary. We have tried a 6-hourly enhancement factor climatology averaged over the same 10 years, and the results are identical to that presented here with a monthly climatology. Note that the Data Wave climatology is derived from simulations with present-day forcing, and may not be robust under climate change with significant shift of the large scale wave patterns (Hemer et al., 2013; Fan et al., 2014). However, as shown in Table 3, its application under moderate change of wind climate is justified . 
The Theory Wave estimate is based on similar ideas. But instead of trying to find a statistical description for the enhancement factor, we started from a statistical description for the surface waves, the empirical wave spectra, and approximated the enhancement factor from that spectrum. The Stokes drift profile is first approximated based on the work of Breivik et al. (2014, 2016), with added directional spreading effects from Webb and Fox-Kemper (2015). The enhancement factor is then calculated from the surface layer averaged Stokes drift, where the boundary layer depth diagnosed from the ocean model is involved. So unlike the Data Wave climatology, the enhancement factor in the Theory Wave estimate is still diagnosed online. But unlike a prognostic wave model, the wave physics is replaced by wave statistics. Note that, following the procedures in Breivik et al. (2016), the Phillips spectrum is used only to determine the shape of the Stokes drift profile. The final profile is constrained by the total Stokes transport, which is estimated from the Pierson-Moskowitz spectrum with a magnitude loss coefficient $c_{1}$ in (19), and the surface Stokes drift, which is assumed to be proportional to the wind with a proportionality constant $c_{2}$ in (23). Both coefficients are determined by matching the Rascle and Ardhuin (2013) global wave hindcast data. No effects from swell are explicitly accounted for, though the approximated mean effect is included by the magnitude loss coefficient. The magnitude loss coefficient used here is consistent with the one reported in Webb and Fox-Kemper (2015) and the resulting enhancement factor agrees reasonably well with that estimated from the full spectrum wave model (see, e.g., Fig. 2d). This insensitivity of the enhancement factor, and thereby the Langmuir mixing effects, to the detailed structure of the Stokes drift profile is partially expected from the LES studies (Harcourt and D'Asaro, 2008; Van Roekel et al., 2012), in which the vertical turbulent kinetic energy collapse into a single curve when the surface layer averaged Stokes drift is used. The Theory Wave approach is also expected to be more robust than the Data Wave one under climate change, as it does not depend on regionally specific empirical parameters, although changes to the swell climate will not be picked up without information from a wave model. 
Overall, the CESM ocean model component appears to be relatively insensitive to the variations of the enhancement factor, showing some tolerance to errors. However, two factors are identified to be important to make both the Data Wave climatology and the Theory Wave estimate a success in parameterizing the Langmuir mixing effects. The first one is the seasonality of the enhancement factor, brought by the effect of Stokes depth versus the boundary layer depth. It accounts for the dependence of the Langmuir mixing effects on the forcing regimes. The smaller enhancement factor in winter seems counterintuitive at first, as ocean surface waves are more vigorous in winter. However, note that the enhancement factor is somehow a correction to the wind effects (to account for the wave effects) on the ocean surface boundary layer vertical mixing. In winter when the boundary layer is deep and dominated by convection, the relative wave effects should not be as large as that in summer, when the wave effects are competing with wind effects and stratification opposes turbulence mixing. The idea is somewhat similar to the modifications proposed by Smyth et al. (2002) on the McWilliams and Sullivan (2000) formulation of the enhancement factor. But here this effect is accounted for by using the surface layer averaged Stokes drift.

The second factor is the effect of misaligned wind and waves, or directional spreading and crossing swell. Swell misaligned with the local wind will reduce the magnitude and change the direction of the Stokes drift (Webb and Fox-Kemper, 2015) and suppress the Langmuir turbulence (Van Roekel et al., 2012). Therefore, the Langmuir mixing effects will generally be overestimated without considering swells. The effect of swells is accounted for in Van Roekel et al. (2012) and LW16 by projecting both the wind and Stokes drift onto the direction of Langmuir cells, assuming that only the component of wind stress parallel to the direction of the Langmuir cells energizes the Langmuir turbulence. The resulting enhancement factor depends on the angle between wind and waves, $\theta_{\mathrm{ww}}$, and the angle between wind and Langmuir cells, $\alpha$, as in (3). As a result, the mean effect of swells is incorporated into Data Wave when averaging the enhancement factor as a whole. In comparison, this effect is only 
partially accounted for in the Theory Wave estimate by allowing a magnitude loss coefficient $c_{1}<1$ in (19) to match the prediction of the total Stokes transport from the Pierson-Moskowitz spectrum with the RA13 wave hindcast data. In addition, setting $c_{1}=1$ will result in an overestimation of the enhancement factor (not shown). Potential improvements are possible by incorporating a higher resolution prognostic wave model with better wave physics. However, the improvements will be limited without considering these two factors.

Note that the Data Wave and the Theory Wave are derived particularly for the Langmuir mixing parameterization. There are other surface wave effects that are not considered here that require better representation, such as the modification of the momentum flux through both a roughened ocean surface and the redistribution of wind-driven momentum across the mixed layer (Sullivan et al., 2004, 2014; Garfinkel et al., 2011; Breivik et al., 2015), the breaking waves (Phillips, 1985; Sullivan et al., 2004, 2007) and interaction between the surface waves and the submesoscale processes (Hamlington et al., 2014; Haney et al., 2015; Suzuki et al., 2016). In cases that these surface wave effects should be considered and parameterized, a prognostic wave model coupled with the climate model is likely required. However, it would be interesting to see whether the effects of these processes could be parameterized statistically.

The statistical modeling approaches explored in this paper were aimed at reproducing the Langmuir mixing effects in LW16 with a much lower computational cost. Of course, computers are continually getting more powerful. Algorithmic developments (e.g., Webb, 2013) may also provide prognostic spectral wave models at a lower computational cost. However, the major cost that is avoided by the theory wave approach developed here is the human cost of implementing a wave model into a coupled model framework. Aside from the modest computational cost (3-10\% of the overall climate model system used here), getting WAVEWATCH III included and vetted in CESM took nearly a decade of work by our team. There are advantages to having a full wave model in a climate model beyond Langmuir mixing (Cavaleri et al., 2012), but the advantage of the Theory Wave is that these improvements can be assessed with 
low computational and human costs before deciding to implement a full wave modeling system.

Finally, it is worth noting that the Langmuir mixing parameterization developed here (Van Roekel et al., 2012), as well as similar ones (McWilliams and Sullivan, 2000; Smyth et al., 2002; Harcourt and D'Asaro, 2008; Reichl et al., 2016; Kantha and Clayson, 2004; Harcourt, 2013; Pearson et al., 2015, etc.), represent incremental improvements over previous non-Langmuir schemes. It may be that a wholly new approach to boundary layer mixing is needed to produce a leap in climate model accuracy. Surely however, errors in other components of the modeling system (atmosphere, mesoscale parameterization, etc.) contribute errors in the ocean boundary layer that are irreducible by direct effort alone.

\section{Conclusion}

In this paper two approximations to the enhancement factor, the Data Wave climatology and the Theory Wave estimation, are explored and tested in CESM as substitutes for the prognostic wave model utilized in the Langmuir mixing parameterization in LW16. Both appear to retain the Langmuir mixing effects brought by coupling with WAVEWATCH III, but with significantly less computational cost (Table 1). The data and source code for both approximations are easily accessible through the CVMix project (Appendix C). The essential equations required in the Theory Wave model are summarized in (25) for quick reference.

Two factors are shown to be important in parameterizing the global Langmuir mixing effects: the seasonality of the enhancement factor, brought by the effect of Stokes depth versus the boundary layer depth, and the effect of misaligned wind and waves, or directional spreading and crossing swell. Both factors help avoiding too much mixing in winter. Without considering these two factors, the potential improvements by incorporating results from higher resolution prognostic wave models with better wave physics will be limited. 


\section{Acknowledgments}

Support for BFK, AW, QL from NSF 1258907, 0934737 and NASA ROSES NNX09AF38G is gratefully acknowledged. This research was made possible by a grant from The Gulf of Mexico Research Initiative. Data are publicly available through the Brown Digital Repository at https://doi.org/10.7301/Z0348H86. We would also like to acknowledge high-performance computing support from Yellowstone (ark:/85065/d7wd3xhc) provided by NCAR's Computational and Information Systems Laboratory, sponsored by the National Science Foundation. NCAR is sponsored by the National Science Foundation. $\varnothing \mathrm{B}$ would like to acknowledge support from CMEMS COPERNICUS through the WAVE2NEMO contract as well as from the Research Council of Norway through the projects RETROSPECT (grant no 244262) and CIRFA (grant 237906). We also appreciate the very helpful comments and suggestions from the four anonymous reviewers.

\section{Appendix A The depth-averaged Stokes drift under a 1D Phillips- type spectrum}

It is useful to know the average Stokes drift over a part of the water column, for example to compute the average Stokes drift experienced by a submerged object. By assuming the 1D Phillips spectrum (7) to be a reasonable approximation to the full spectrum, it is possible to provide a closed-form expression for the integral of Eq (8), i.e., the Stokes drift transport between the vertical level $z_{0}$ and the surface. The Stokes transport under Eq (8) can be written

$$
V\left(y_{0}\right)=\frac{u_{0}^{\mathrm{S}}}{2 k_{\mathrm{p}}} \int_{y_{0}}^{0} \mathrm{e}^{-y} d y-\beta \sqrt{\pi} \underbrace{\int_{y_{0}}^{0} \sqrt{y} \operatorname{erfc}(\sqrt{y})}_{I_{1}} d y .
$$

Here we have introduced the variable substitution $y=-2 k_{\mathrm{p}} z$ (also applies to variables with subscript 0 ) and $u_{0}^{\mathrm{S}}$ is the surface Stokes drift speed. The integral $I_{1}$ can be solved by first substituting $w=\sqrt{y}$,

$$
I_{1}=2 \int_{0}^{w_{0}} w^{2} \operatorname{erfc} w d w .
$$


Then, integrating by parts gives

$$
I_{1}=\frac{2}{3} w_{0}^{3} \operatorname{erfc} w_{0}+\frac{4}{3 \sqrt{\pi}} \underbrace{\int_{0}^{w_{0}} w^{3} \mathrm{e}^{-w^{2}} d w}_{I_{2}} .
$$

941 The integral $I_{2}$ can be found analytically (see Eq (3.321.6) of Gradshteyn and ${ }_{942}$ Ryzhik 2007),

$$
I_{2}=1-\left(1+w_{0}^{2}\right) \mathrm{e}^{-w_{0}^{2}} .
$$

${ }_{943} \mathrm{Eq}$ (A.1) can now be solved,

$$
V\left(y_{0}\right)=\frac{u_{\mathrm{Phil}}^{0}}{2 k_{\mathrm{p}}}\left\{1-\mathrm{e}^{-y_{0}}-\frac{2 \beta}{3}\left[1+\sqrt{\pi} y_{0}^{3 / 2} \operatorname{erfc} \sqrt{y_{0}}-\left(1+y_{0}\right) \mathrm{e}^{-y_{0}}\right]\right\} .
$$

${ }_{944}$ Reverting back to dimensional variables we get

$$
\begin{aligned}
V\left(z_{0}\right)= & \frac{u_{0}^{\mathrm{S}}}{2 k_{\mathrm{p}}}\left\{1-\mathrm{e}^{-2 k_{\mathrm{p}}\left|z_{0}\right|}-\frac{2 \beta}{3}\left[1+\sqrt{\pi}\left(2 k_{\mathrm{p}}\left|z_{0}\right|\right)^{3 / 2} \operatorname{erfc}\left(\sqrt{2 k_{\mathrm{p}}\left|z_{0}\right|}\right)\right.\right. \\
& \left.\left.-\left(1+2 k_{\mathrm{p}}\left|z_{0}\right|\right) \mathrm{e}^{-2 k_{\mathrm{p}}\left|z_{0}\right|}\right]\right\} .
\end{aligned}
$$

945 In order to find the average Stokes drift between a lower level $z_{0}$ and an 946 upper level $z_{1}$ all that is needed is to use Eq (A.6) twice to find

$$
\bar{u}_{0}^{\mathrm{S}}=\frac{V\left(z_{0}\right)-V\left(z_{1}\right)}{z_{1}-z_{0}} .
$$

\footnotetext{
947 Appendix B Wave directional spreading refinement for a 1D Phillipstype spectrum

\section{B.1 Depth-dependent Stokes drift}

950

The depth-dependent Stokes drift for a 1D Phillips-type spectrum (Breivik et al., 2016) can be modified to include wave directional spreading effects by using the Padé-approximated, DHH directional-Stokes-drift-component from
} 
953

$$
\begin{aligned}
\boldsymbol{u}_{\text {Phil-s }}^{\mathrm{S}}(z)= & \hat{\boldsymbol{e}}^{\mathrm{w}} \frac{2}{g} \int_{0}^{\infty} H_{\mathrm{DHH}}\left(\omega / \omega_{\mathrm{p}}\right) \omega^{3} S_{\mathrm{Phil}}(\omega) \mathrm{e}^{2 k z} \mathrm{~d} \omega \\
= & \hat{\boldsymbol{e}}^{\mathrm{w}} 2 \alpha g \int_{\omega_{\mathrm{p}}}^{\infty} H_{\mathrm{DHH}}\left(\omega / \omega_{\mathrm{p}}\right) \omega^{-2} \mathrm{e}^{2 z \omega^{2} / g} \mathrm{~d} \omega \\
= & \hat{\boldsymbol{e}}^{\mathrm{w}} 2 \alpha g\left\{\int_{\omega_{\mathrm{p}}}^{1.6 \omega_{\mathrm{p}}}\left(\frac{0.98-0.19\left(\omega / \omega_{\mathrm{p}}\right)+0.0058\left(\omega / \omega_{\mathrm{p}}\right)^{2}}{1-0.26\left(\omega / \omega_{\mathrm{p}}\right)+0.12\left(\omega / \omega_{\mathrm{p}}\right)^{2}}\right) \omega^{-2} \mathrm{e}^{2 z \omega^{2} / g} \mathrm{~d} \omega\right. \\
& \left.+\int_{1.6 \omega_{\mathrm{p}}}^{\infty} 0.777 \omega^{-2} \mathrm{e}^{2 z \omega^{2} / g} \mathrm{~d} \omega\right\} .
\end{aligned}
$$

${ }_{954} \quad$ Instead of evaluating $\boldsymbol{u}_{\text {Phil-s }}^{\mathrm{S}}$ directly for the first interval, it is much easier 955 to work with an approximation of the Padé-approximant. For $r=\omega / \omega_{\mathrm{p}}$ and ${ }_{956} a_{0}, a_{2}>0$ ( $a_{1}$ arbitrary), let

$$
f(r)=a_{0}+a_{1} r-a_{2} r^{2}
$$

957 such that following conditions hold:

$$
\begin{aligned}
f(1) & =H_{\mathrm{DHH}}(1), \\
f(1.6) & =H_{\mathrm{DHH}}(1.6), \\
\int_{1}^{1.6} f(r) \mathrm{d} r & \approx \int_{1}^{1.6} H_{\mathrm{DHH}}(r) \mathrm{d} r .
\end{aligned}
$$

958 One possible solution is to let $a_{2}=0.0958, a_{1}=0$ and $a_{0}=1.021$. Then ${ }_{959}$ (B.2) holds to within a relative error of $1.1 \times 10^{-3}$ (in the interval) of the Padé960 approximant.

961 Then (B.1) can be written as

$$
\begin{aligned}
\boldsymbol{u}_{\text {Phil-s }}^{\mathrm{S}}(z)= & \hat{\boldsymbol{e}}^{\mathrm{w}} 2 \alpha g\left\{\int_{\omega_{\mathrm{p}}}^{1.6 \omega_{\mathrm{p}}}\left[\left(a_{0}-0.777\right) \omega^{-2}-\frac{a_{2}}{\omega_{\mathrm{p}}^{2}}\right] \mathrm{e}^{2 z \omega^{2} / g} \mathrm{~d} \omega\right. \\
& \left.+0.777 \int_{\omega_{\mathrm{p}}}^{\infty} \omega^{-2} \mathrm{e}^{2 z \omega^{2} / g} \mathrm{~d} \omega\right\}
\end{aligned}
$$


At the surface $(z=0)$, this simplifies to

$$
\begin{aligned}
u_{0, \text { Phil-s }}^{\mathrm{S}} & =\left|\boldsymbol{u}_{\text {Phil-s }}^{\mathrm{S}}(0)\right| \\
& =u_{0, \text { Phil }}^{\mathrm{S}}\left\{\int_{\omega_{\mathrm{p}}}^{1.6 \omega_{\mathrm{p}}}\left[\left(a_{0}-0.777\right) \omega_{\mathrm{p}} \omega^{-2}-\frac{a_{2}}{\omega_{\mathrm{p}}}\right] \mathrm{d} \omega+0.777\right\} \\
& \approx 0.811 u_{0, \text { Phil }}^{\mathrm{S}},
\end{aligned}
$$

963

$$
\begin{aligned}
\boldsymbol{u}_{\text {Phil-s }}^{\mathrm{S}}(z)= & \hat{\boldsymbol{e}}^{\mathrm{w}} \frac{2 \alpha g}{\omega_{\mathrm{p}}}\left\{a_{0}\left[T_{1}\left(k_{\mathrm{p}}, z\right)-\left(1+\frac{a_{2}}{a_{0}} \frac{1}{4 k_{\mathrm{p}}|z|}\right) T_{2}\left(k_{\mathrm{p}}, z\right)\right]\right. \\
& \left.-\frac{a_{0}-0.777}{1.6}\left[T_{1}\left(k_{\mathrm{p}}^{*}, z\right)-\left(1+\frac{1}{a_{0}-0.777} \frac{1.6^{2}}{4 k_{\mathrm{p}}^{*}|z|}\right) T_{2}\left(k_{\mathrm{p}}^{*}, z\right)\right]\right\} .
\end{aligned}
$$

${ }_{967}$ To further simplify, let

$$
\begin{aligned}
& B_{1}(z)=1+\frac{0.0469}{2 k_{\mathrm{p}}|z|} \approx 1+\frac{a_{2}}{a_{0}} \frac{1}{4 k_{\mathrm{p}}|z|}, \\
& B_{2}(z)=1+\frac{0.503}{2 k_{\mathrm{p}}^{*}|z|} \approx 1+\frac{1}{a_{0}-0.777} \frac{1.6^{2}}{4 k_{\mathrm{p}}^{*}|z|} .
\end{aligned}
$$

${ }_{968}$ Then (B.7) can be rewritten as

$$
\begin{aligned}
\boldsymbol{u}_{\text {Phil-s }}^{\mathrm{S}}(z)= & \hat{\boldsymbol{e}}^{\mathrm{w}} u_{0, \text { Phil }}^{\mathrm{S}}\left\{a_{0}\left[T_{1}\left(k_{\mathrm{p}}, z\right)-B_{1}(z) T_{2}\left(k_{\mathrm{p}}, z\right)\right]\right. \\
& \left.-\frac{a_{0}-0.777}{1.6}\left[T_{1}\left(k_{\mathrm{p}}^{*}, z\right)-B_{2}(z) T_{2}\left(k_{\mathrm{p}}^{*}, z\right)\right]\right\} \\
\approx & \hat{\boldsymbol{e}}^{\mathrm{w}} u_{0, \text { Phil-s }}^{\mathrm{S}}\left\{1.26\left[T_{1}\left(k_{\mathrm{p}}, z\right)-B_{1}(z) T_{2}\left(k_{\mathrm{p}}, z\right)\right]\right. \\
& \left.-0.188\left[T_{1}\left(k_{\mathrm{p}}^{*}, z\right)-B_{2}(z) T_{2}\left(k_{\mathrm{p}}^{*}, z\right)\right]\right\} .
\end{aligned}
$$


972

$$
\begin{aligned}
J_{1}(k) & =\int_{z_{0}}^{0} T_{1}(k, z) \mathrm{d} z=\frac{1}{2 k}\left(1-T_{1}\left(k, z_{0}\right)\right), \\
J_{2}(k) & =\int_{z_{0}}^{0} T_{2}(k, z) \mathrm{d} z \\
& =\frac{2\left|z_{0}\right|}{3} T_{2}\left(k, z_{0}\right)+\frac{1}{3 k}-\left(\frac{1}{3 k}+\frac{2\left|z_{0}\right|}{3}\right) T_{1}\left(k, z_{0}\right), \\
J_{3}(k) & =\int_{z_{0}}^{0} \frac{1}{2 k|z|} T_{2}(k, z) \mathrm{d} z=\frac{1}{k}\left[1+T_{2}\left(k, z_{0}\right)-T_{1}\left(k, z_{0}\right)\right] .
\end{aligned}
$$

973 See also Appendix A for the 1D profile without spreading. Substituting (B.12) 974 - (B.14) back into (B.11), we get,

$$
\begin{aligned}
\left\langle\boldsymbol{u}_{\text {Phil-s }}^{\mathrm{S}}\right\rangle_{z_{0}} \approx & \hat{\boldsymbol{e}}^{\mathrm{w}} u_{0, \text { Phil-s }}^{\mathrm{S}}\{0.715 \\
& +\left(\frac{0.151}{k_{\mathrm{p}}\left|z_{0}\right|}-0.840\right)\left[1-T_{1}\left(k_{\mathrm{p}}, z_{0}\right)\right] \\
& -\left(0.840+\frac{0.0591}{k_{\mathrm{p}}\left|z_{0}\right|}\right) T_{2}\left(k_{\mathrm{p}}, z_{0}\right) \\
& +\left(\frac{0.0632}{k_{\mathrm{p}}^{*}\left|z_{0}\right|}+0.125\right)\left[1-T_{1}\left(k_{\mathrm{p}}^{*}, z_{0}\right)\right] \\
& \left.+\left(0.125+\frac{0.0946}{k_{\mathrm{p}}^{*}\left|z_{0}\right|}\right) T_{2}\left(k_{\mathrm{p}}^{*}, z_{0}\right)\right\} .
\end{aligned}
$$




\section{B.3 Total Stokes transport}

The total Stokes transport is derived by integrating (B.10) from $z=-\infty$ to $z=0$. Notice that

$$
\begin{aligned}
\int_{-\infty}^{0} T_{1}(k, z) \mathrm{d} z & =\frac{1}{2 k}, \\
\int_{-\infty}^{0} T_{2}(k, z) \mathrm{d} z & =\frac{1}{3 k}, \\
\int_{-\infty}^{0} \frac{1}{2 k|z|} T_{2}(k, z) \mathrm{d} z & =\frac{1}{k} .
\end{aligned}
$$

The total Stokes transport is then

$$
\begin{aligned}
\boldsymbol{V}_{\text {Phil-s }}^{\mathrm{S}}= & \int_{-\infty}^{0} \boldsymbol{u}_{\text {Phil-s }}^{\mathrm{S}}(z) \mathrm{d} z \\
= & \hat{\boldsymbol{e}}^{\mathrm{w}} u_{0, \text { Phil-s }}^{\mathrm{S}}\left[1.26\left(\frac{1}{2 k_{\mathrm{p}}}-\frac{1}{3 k_{\mathrm{p}}}-\frac{0.0469}{k_{\mathrm{p}}}\right)\right. \\
& \left.-0.188\left(\frac{1}{k_{\mathrm{p}}^{*}}-\frac{1}{3 k_{\mathrm{p}}^{*}}-\frac{0.503}{k_{\mathrm{p}}^{*}}\right)\right] \\
\approx & \hat{\boldsymbol{e}}^{\mathrm{w}} \frac{0.176 u_{0, \text { Phil-s }}^{\mathrm{S}}}{k_{\mathrm{p}}} .
\end{aligned}
$$

\section{Appendix C The Data Wave and Theory Wave in CVMix}

with the four arguments being the 10-meter wind, the water side surface friction velocity, the boundary layer depth, and a global parameter of the type cvmix_global_params_type, respectively, all with SI units. This function returns the enhancement factor and should be called before passing it to the subroutines cvmix_kpp_compute_turbulent_scales and cvmix_coeffs_kpp.

The Data Wave requires file I/O and therefore is suggested to be implemented in the ocean model outside the CVMix framework. However, for testing purpose, another public function is available in a fork of CVMix (https://github.com/qingli411/CVMix-src), 
cvmix_kpp_efactor_read(infile, lon, lat, time)

with the four arguments being the filename of the input enhancement factor data, longitude, latitude and day of a year to be interpolated. The monthly enhancement factor climatology data is saved in Fortran binary format for the application considered here as well as in netCDF format for more general applications, all of which are available in the Brown Digital Repository (https://doi.org/10.7301/Z0348H86).

\section{References}

Ardhuin, F., Marié, L., Rascle, N., Forget, P., Roland, A., 2009. Observation and Estimation of Lagrangian, Stokes, and Eulerian Currents Induced by Wind and Waves at the Sea Surface. Journal of Physical Oceanography 39 (11), $2820-2838$.

Ardhuin, F., Rascle, N., Belibassakis, K. A., 2008. Explicit wave-averaged primitive equations using a generalized Lagrangian mean. Ocean Modelling 20 (1), $35-60$.

Belcher, S. E., Grant, A. L. M., Hanley, K. E., Fox-Kemper, B., Van Roekel, L., Sullivan, P. P., Large, W. G., Brown, A., Hines, A., Calvert, D., Rutgersson, A., Pettersson, H., Bidlot, J.-R., Janssen, P. A. E. M., Polton, J. A., 2012. A global perspective on Langmuir turbulence in the ocean surface boundary layer. Geophysical Research Letters 39 (18), L18605.

Bennetts, L. G., Squire, V. A., 2012. On the calculation of an attenuation coefficient for transects of ice-covered ocean. Proc. R. Soc. A 468 (2137), $136-162$.

Bouws, E., 1998. Guide to wave analysis and forcasting. Tech. Rep. 702, World Meteorological Organization.

Breivik, O., Bidlot, J.-R., Janssen, P. A. E. M., 2016. A Stokes drift approximation based on the Phillips spectrum. Ocean Modelling 100, 49-56. 
Breivik, O., Janssen, P. A. E. M., Bidlot, J.-R., 2014. Approximate Stokes Drift Profiles in Deep Water. Journal of Physical Oceanography 44 (9), 2433-2445.

Breivik, O., Mogensen, K., Bidlot, J.-R., Balmaseda, M. A., Janssen, P. a. E. M., 2015. Surface wave effects in the NEMO ocean model: Forced and coupled experiments. Journal of Geophysical Research 120 (4), 2973-2992.

Cavaleri, L., Fox-Kemper, B., Hemer, M., 2012. Wind waves in the coupled climate system. Bulletin of the American Meteorological Society 93 (11), 16511661.

Craik, A. D. D., Leibovich, S., 1976. A rational model for Langmuir circulations. Journal of Fluid Mechanics 73 (03), 401-426.

Danabasoglu, G., Bates, S. C., Briegleb, B. P., Jayne, S. R., Jochum, M., Large, W. G., Peacock, S., Yeager, S. G., 2012. The CCSM4 Ocean Component. Journal of Climate 25 (5), 1361-1389.

D'Asaro, E. A., 2001. Turbulent Vertical Kinetic Energy in the Ocean Mixed Layer. Journal of Physical Oceanography 31 (12), 3530-3537.

D'Asaro, E. A., Thomson, J., Shcherbina, A. Y., Harcourt, R. R., Cronin, M. F., Hemer, M. A., Fox-Kemper, B., 2014. Quantifying upper ocean turbulence driven by surface waves. Geophysical Research Letters 41 (1), 102-107.

de Boyer Montégut, C., Madec, C., Fischer, A. S., Lazar, A., Iudicone, D., 2004. Mixed layer depth over the global ocean: An examination of profile data and a profile-based climatology. Journal of Geophysical Research 109 (C12), C12003.

Donelan, M. A., Hamilton, J., Hui, W. H., 1985. Directional spectra of windgenerated waves. Philosophical Transactions of the Royal Society A: Mathematical, Physical and Engineering Sciences 315, 509-562.

Fan, Y., Griffies, S. M., 2014. Impacts of parameterized langmuir turbulence and nonbreaking wave mixing in global climate simulations. Journal of Climate 27 (12), 4752-4775. 
Fan, Y., Lin, S.-J., Griffies, S. M., Hemer, M. A., 2014. Simulated Global Swell and Wind-Sea Climate and Their Responses to Anthropogenic Climate Change at the End of the Twenty-First Century. Journal of Climate 27 (10), $3516-3536$.

Feddersen, F., 2004. Effect of wave directional spread on the radiation stress: Comparing theory and observations. Coastal Engineering 51 (5-6), 473-481.

Fox-Kemper, B., Danabasoglu, G., Ferrari, R., Griffies, S. M., Hallberg, R. W., Holland, M. M., Maltrud, M. E., Peacock, S., Samuels, B. L., 2011. Parameterization of mixed layer eddies. III: Implementation and impact in global ocean climate simulations. Ocean Modelling 39 (1-2), 61-78.

Garfinkel, C. I., Molod, a. M., Oman, L. D., Song, I.-S., 2011. Improvement of the GEOS-5 AGCM upon updating the air-sea roughness parameterization. Geophysical Research Letters 38 (18), L18702.

Gradshteyn, I., Ryzhik, I., 2007. Table of Integrals, Series, and Products, 7th edition. Academic Press, London.

Grant, A. L. M., Belcher, S. E., 2009. Characteristics of Langmuir turbulence in the ocean mixed layer. Journal of Physical Oceanography 39 (8), 1871-1887.

Griffies, S. M., Biastoch, A., Böning, C., Bryan, F., Danabasoglu, G., Chassignet, E. P., England, M. H., Gerdes, R., Haak, H., Hallberg, R. W., Hazeleger, W., Jungclaus, J., Large, W. G., Madec, G., Pirani, A., Samuels, B. L., Scheinert, M., Gupta, A. S., Severijns, C. A., Simmons, H. L., Treguier, A. M., Winton, M., Yeager, S., Yin, J., 2009. Coordinated Ocean-ice Reference Experiments (COREs). Ocean Modelling 26 (1-2), 1-46.

Hamlington, P. E., Van Roekel, L. P., Fox-Kemper, B., Julien, K., Chini, G. P., 2014. Langmuir-submesoscale interactions: Descriptive analysis of multiscale frontal spindown simulations. Journal of Physical Oceanography 44 (9), 2249 -2272 . 
Haney, S., Fox-Kemper, B., Julien, K., Webb, A., 2015. Symmetric and geostrophic instabilities in the wave-forced ocean mixed layer. Journal of Physical Oceanography 45 (12), 3033-3056.

Hanley, K. E., Belcher, S. E., Sullivan, P. P., 2010. A global climatology of wind-wave interaction. Journal of Physical Oceanography 40 (6), 1263-1282.

Harcourt, R. R., 2013. A second-moment closure model of Langmuir turbulence. Journal of Physical Oceanography 43 (4), 673-697.

Harcourt, R. R., D'Asaro, E. A., 2008. Large-eddy simulation of Langmuir turbulence in pure wind seas. Journal of Physical Oceanography 38 (7), 15421562.

Hasselmann, K., Barnett, T., Bouws, E., Carlson, H., Cartwright, D. E., Enke, K., Ewing, J. A., Gienapp, H., Hasselmann, D. E., Kruseman, P., Meerburg, A., Muller, P., Olbers, D. J., Richter, K., Sell, W., Walden, H., 1973. Measurements of wind-wave growth and swell decay during the Joint North Sea Wave Project (JONSWAP). Tech. Rep. 8 0, Deutsches Hydrographisches Institut, Hamburg.

Hemer, M. A., Fan, Y., Mori, N., Semedo, A., Wang, X. L., 2013. Projected changes in wave climate from a multi-model ensemble. Nature Climate Change 3 (5), 471-476.

Holthuijsen, L. H., 2007. Waves in Oceanic and Coastal Waters. Cambridge University Press, New York.

Huang, C. J., Qiao, F., 2010. Wave-turbulence interaction and its induced mixing in the upper ocean. Journal of Geophysical Research: Oceans 115 (C4), C04026.

Kantha, L. H., Clayson, C. A., 2004. On the effect of surface gravity waves on mixing in the oceanic mixed layer. Ocean Modelling 6 (2), 101-124. 
Kenyon, K. E., 1969. Stokes drift for random gravity waves. Journal of Geophysical Research 74 (28), 6991.

Key, R. M., Kozyr, A., Sabine, C. L., Lee, K., Wanninkhof, R., Bullister, J. L., a. Feely, R., Millero, F. J., Mordy, C., Peng, T.-H., 2004. A global ocean carbon climatology: Results from Global Data Analysis Project (GLODAP). Global Biogeochemical Cycles 18, GB4031.

Kukulka, T., Plueddemann, A. J., Trowbridge, J. H., Sullivan, P. P., 2009. Significance of Langmuir circulation in upper ocean mixing: Comparison of observations and simulations. Geophysical Research Letters 36 (10), L10603.

Large, W. G., Mcwilliams, J. C., Doney, S. C., 1994. Oceanic vertical mixing: A review and a model with a nonlocal boundary layer parameterization. Reviews of Geophysics 32 (4), 363-403.

Large, W. G., Yeager, S. G., 2009. The global climatology of an interannually varying air-sea flux data set. Climate Dynamics 33 (2-3), 341-364.

Levitus, S., Boyer, T., Concright, M., Johnson, D., O’Brien, T., Antonov, J., Stephens, C., Garfield, R., 1998. World Ocean Database 1998: Volume 1: Introduction. Tech. Rep. Atlas NESDIS18, NOAA.

Li, M., Garrett, C., Skyllingstad, E. D., 2005. A regime diagram for classifying turbulent large eddies in the upper ocean. Deep-Sea Research Part I: Oceanographic Research Papers 52 (2), 259-278.

Li, M., Zahariev, K., Garrett, C., 1995. Role of Langmuir circulation in the deepening of the ocean surface mixed layer. Science, 1955-1957.

Li, Q., Webb, A., Fox-Kemper, B., Craig, A., Danabasoglu, G., Large, W. G., Vertenstein, M., 2016. Langmuir mixing effects on global climate: WAVEWATCH III in CESM. Ocean Modelling 103, 145-160.

McWilliams, J. C., Huckle, E., Liang, J.-H., Sullivan, P. P., 2014. Langmuir turbulence in swell. Journal of Physical Oceanography 44 (3), 870-890. 
McWilliams, J. C., Restrepo, J. M., 1999. The wave-driven ocean circulation. Journal of Physical Oceanography 29, 2523-2540.

McWilliams, J. C., Sullivan, P. P., 2000. Vertical mixing by Langmuir circulations. Spill Science and Technology Bulletin 6 (3), 225-237.

McWilliams, J. C., Sullivan, P. P., Moeng, C.-H., 1997. Langmuir turbulence in the ocean. Journal of Fluid Mechanics 334 (1), 1-30.

Myrhaug, D., 2013. Some statistical aspects of wave-induced drift in sea states. Coastal Engineering 78 (0378), 53-56.

Myrhaug, D., 2015. Stokes drift estimation based on long-term variation of wave conditions. Proceedings of the Institution of Mechanical Engineers, Part M: Journal of Engineering for the Maritime Environment 229 (2), 141-146.

Pearson, B. C., Grant, A. L. M., Polton, J. A., Belcher, S. E., 2015. Langmuir turbulence and surface heating in the ocean surface boundary layer. Journal of Physical Oceanography 45, 2897-2911.

Phillips, O. M., 1958. The equilibrium range in the spectrum of wind-generated waves. Journal of Fluid Mechanics 4 (04), 426.

Phillips, O. M., 1985. Spectral and statistical properties of the equilibrium range in wind-generated gravity waves. Journal of Fluid Mechanics 156, 505-531.

Pierson, W. J., Moskowitz, L., 1964. A proposed spectral form for fully developed wind seas based on the similarity theory of S. A. Kitaigorodskii. Journal of Geophysical Research 69 (24), 5181-5190.

Pierson, W. J., Neumann, G., James, R. W., 1955. Practical Methods for Observing and Forecasting Ocean Waves by Means of Wave Spectra and Statistics. US Navy Hydrographic Office.

Rascle, N., Ardhuin, F., 2013. A global wave parameter database for geophysical applications. Part 2: Model validation with improved source term parameterization. Ocean Modelling 70, 174-188. 
Rascle, N., Ardhuin, F., Queffeulou, P., Croizé-Fillon, D., 2008. A global wave parameter database for geophysical applications. Part 1: Wavecurrent-turbulence interaction parameters for the open ocean based on traditional parameterizations. Ocean Modelling 25 (3-4), 154-171.

Reichl, B. G., Wang, D., Hara, T., Ginis, I., Kukulka, T., 2016. Langmuir Turbulence Parameterization in Tropical Cyclone Conditions. Journal of Physical Oceanography 46 (3), 863-886.

Shields, C. A., Bailey, D. A., Danabasoglu, G., Jochum, M., Kiehl, J. T., Levis, S., Park, S., 2012. The Low-Resolution CCSM4. Journal of Climate 25 (12), 3993-4014.

Skyllingstad, E. D., Denbo, D. W., 1995. An ocean large-eddy simulation of Langmuir circulations and convection in the surface mixed layer. Journal of Geophysical Research 100 (C5), 8501.

Smyth, W. D., Skyllingstad, E. D., Crawford, G. B., Wijesekera, H., 2002. Nonlocal fluxes and Stokes drift effects in the K-profile parameterization. Ocean Dynamics 52 (3), 104-115.

Squire, V. A., Moore, S. C., 1980. Direct measurement of the attenuation of ocean waves by pack ice. Nature 283 (5745), 365-368.

Steele, M., Morley, R., Ermold, W., 2001. PHC: A global ocean hydrography with a high-quality Arctic Ocean. Journal of Climate 14, 2079-2087.

Sullivan, P. P., McWilliams, J. C., Melville, W. K., 2004. The oceanic boundary layer driven by wave breaking with stochastic variability. Part 1 . Direct numerical simulations. Journal of Fluid Mechanics 507, 143-174.

Sullivan, P. P., McWilliams, J. C., Melville, W. K., 2007. Surface gravity wave effects in the oceanic boundary layer: large-eddy simulation with vortex force and stochastic breakers. Journal of Fluid Mechanics 593, 405-452. 
Sullivan, P. P., McWilliams, J. C., Patton, E. G., 2014. Large-eddy simulation of marine atmospheric boundary layers above a spectrum of moving waves. Journal of the Atmospheric Sciences 71 (11), 4001-4027.

Suzuki, N., Fox-Kemper, B., 2016. Understanding Stokes forces in the waveaveraged equations. Journal of Geophysical Research: Oceans 121 (5), 35793596.

Suzuki, N., Fox-Kemper, B., Hamlington, P. E., Van Roekel, L. P., 2016. Surface waves affect frontogenesis. Journal of Geophysical Research: Oceans 121 (5), $3597-3624$.

Teixeira, M. A. C., 2011. A linear model for the structure of turbulence beneath surface water waves. Ocean Modelling 36 (1-2), 149-162.

Tennekes, H., Lumley, J. L., 1972. A First Course in Turbulence. The MIT Press, Cambridge, MA.

Thorpe, S. A., 2007. An Introduction to Ocean Turbulence. Cambridge University Press, New York.

Thorpe, S. A., Osborn, T. R., Jackson, J. F. E., Hall, A. J., Lueck, R. G., 2003. Measurements of Turbulence in the Upper-Ocean Mixing Layer Using Autosub. Journal of Physical Oceanography 33 (1), 122-145.

Tolman, H. L., 2009. User manual and system documentation of WAVEWATCH III version 3.14. Tech. Rep. 276, NOAA / NWS / NCEP / MMAB.

Tseng, R.-S., D'Asaro, E. A., 2004. Measurements of turbulent vertical kinetic energy in the ocean mixed layer from Lagrangian floats. Journal of Physical Oceanography 34 (9), 1984-1990.

Van Roekel, L., Fox-Kemper, B., Sullivan, P. P., Hamlington, P. E., Haney, S. R., 2012. The form and orientation of Langmuir cells for misaligned winds and waves. Journal of Geophysical Research 117 (C05001), C05001. 
Webb, A., Fox-Kemper, B., 2011. Wave spectral moments and Stokes drift estimation. Ocean Modelling 40 (3-4), 273-288.

Webb, A., Fox-Kemper, B., 2015. Impacts of wave spreading and multidirectional waves on estimating Stokes drift. Ocean Modelling 96, Part 1, 49-64.

Webb, A. A., 2013. Stokes Drift and Meshless Wave Modeling. Ph.D. thesis, University of Colorado.

URL http://www.geo.brown.edu/research/Fox-Kemper/pubs/pdfs/ WebbThesis.pdf

Weller, R. A., Price, J. F., 1988. Langmuir circulation within the oceanic mixed layer. Deep Sea Research Part A. Oceanographic Research Papers 35 (5), $711-747$.

Williams, T. D., Bennetts, L. G., Squire, V. A., Dumont, D., Bertino, L., 2013. Wave-ice interactions in the marginal ice zone. Part 1: Theoretical foundations. Ocean Modelling 71, 81-91. 


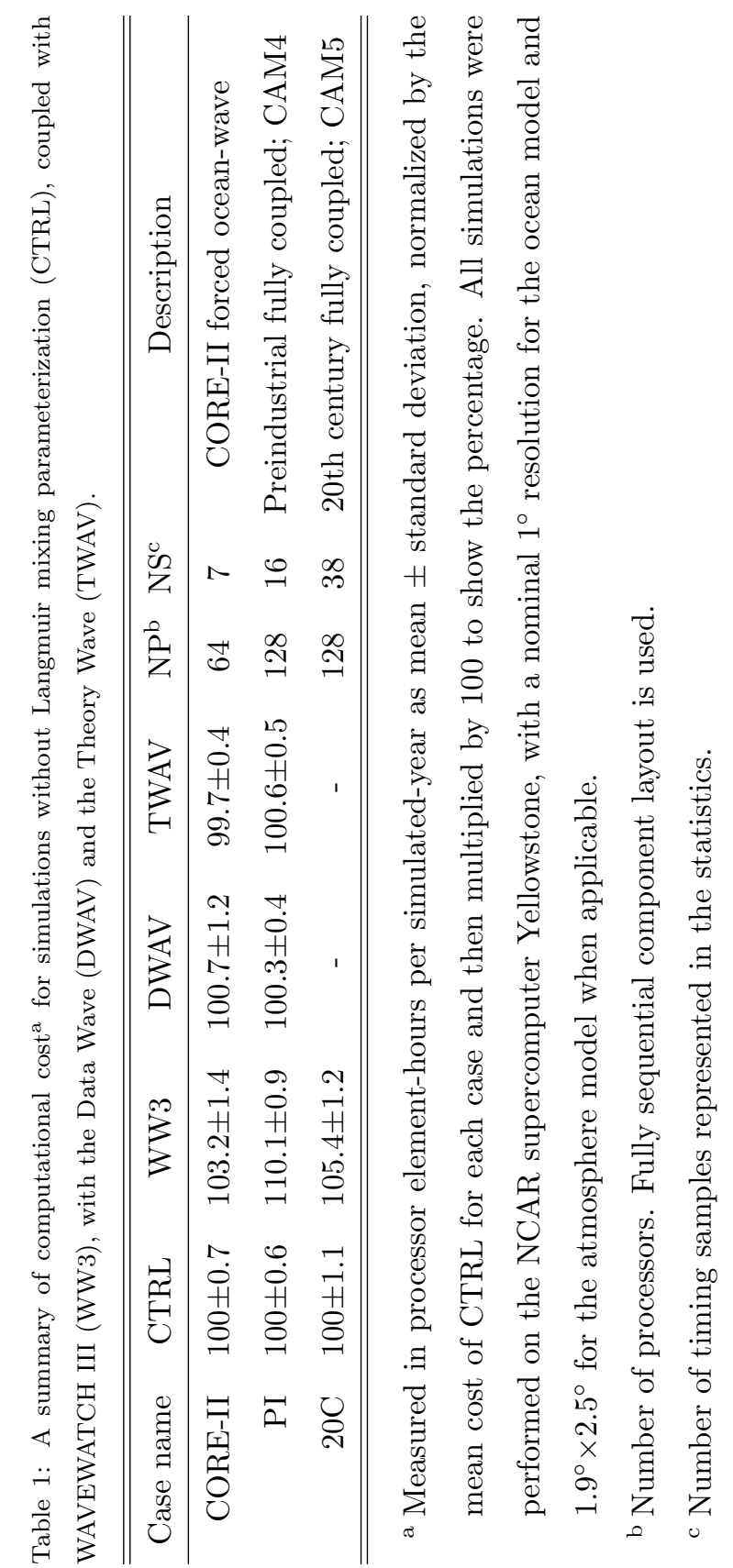




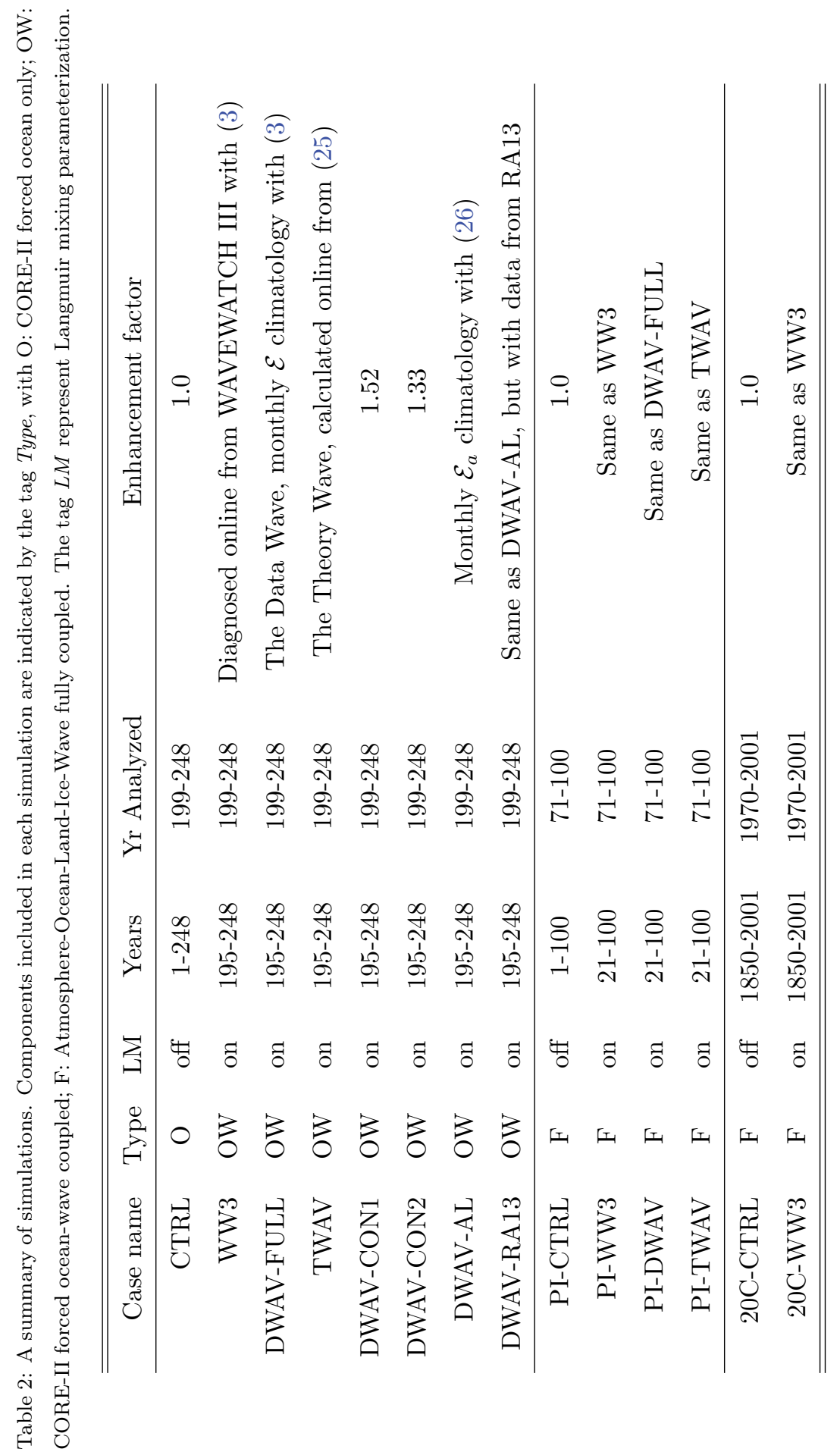


Table 3: Comparison of the 10-year averaged wave statistics over the last 10 years (denoted as $T_{2}$; corresponding to forcing years 2000-2009) versus the first 10 years ( $T_{1}$; corresponding to forcing years 1960-1969) of the 50-year data. See text for more details.

\begin{tabular}{rcccccc}
\hline \hline Case & Measure & $\Delta U_{10}(\%)$ & $\Delta u^{*}(\%)$ & $\Delta H_{\mathrm{m}_{0}}(\%)$ & $\Delta u_{0}^{\mathrm{S}}(\%)$ & $\Delta \mathcal{E}(\%)$ \\
\hline Global & $\mathrm{NMD}^{\mathrm{a}}$ & 2.5 & 3.4 & 3.9 & 3.8 & 0.0 \\
& $\mathrm{NRMSD}^{\mathrm{b}}$ & 10.0 & 12.3 & 9.1 & 15.7 & 2.8 \\
\hline $90^{\circ} \mathrm{S}-30^{\circ} \mathrm{S}$ & $\mathrm{NMD}$ & 5.3 & 7.0 & 7.9 & 7.8 & 0.3 \\
& $\mathrm{NRMSD}$ & 8.5 & 10.9 & 10.6 & 12.2 & 2.4 \\
\hline $30^{\circ} \mathrm{S}-30^{\circ} \mathrm{N}$ & $\mathrm{NMD}$ & 0.5 & 0.4 & 0.9 & 0.3 & -0.1 \\
& $\mathrm{NRMSD}$ & 11.4 & 13.9 & 8.6 & 18.4 & 3.0 \\
\hline $30^{\circ} \mathrm{N}-90^{\circ} \mathrm{N}$ & $\mathrm{NMD}$ & 1.2 & 2.0 & 1.1 & 1.9 & -0.2 \\
& $\mathrm{NRMSD}$ & 6.0 & 7.7 & 7.3 & 9.3 & 2.4 \\
\hline \hline
\end{tabular}

a Normalized Mean Difference: $\frac{\overline{x\left(T_{2}\right)}-\overline{x\left(T_{1}\right)}}{\overline{x\left(T_{1}\right)}} \times 100 \%$

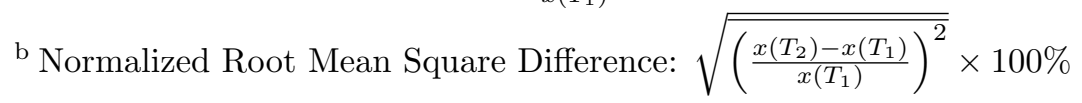



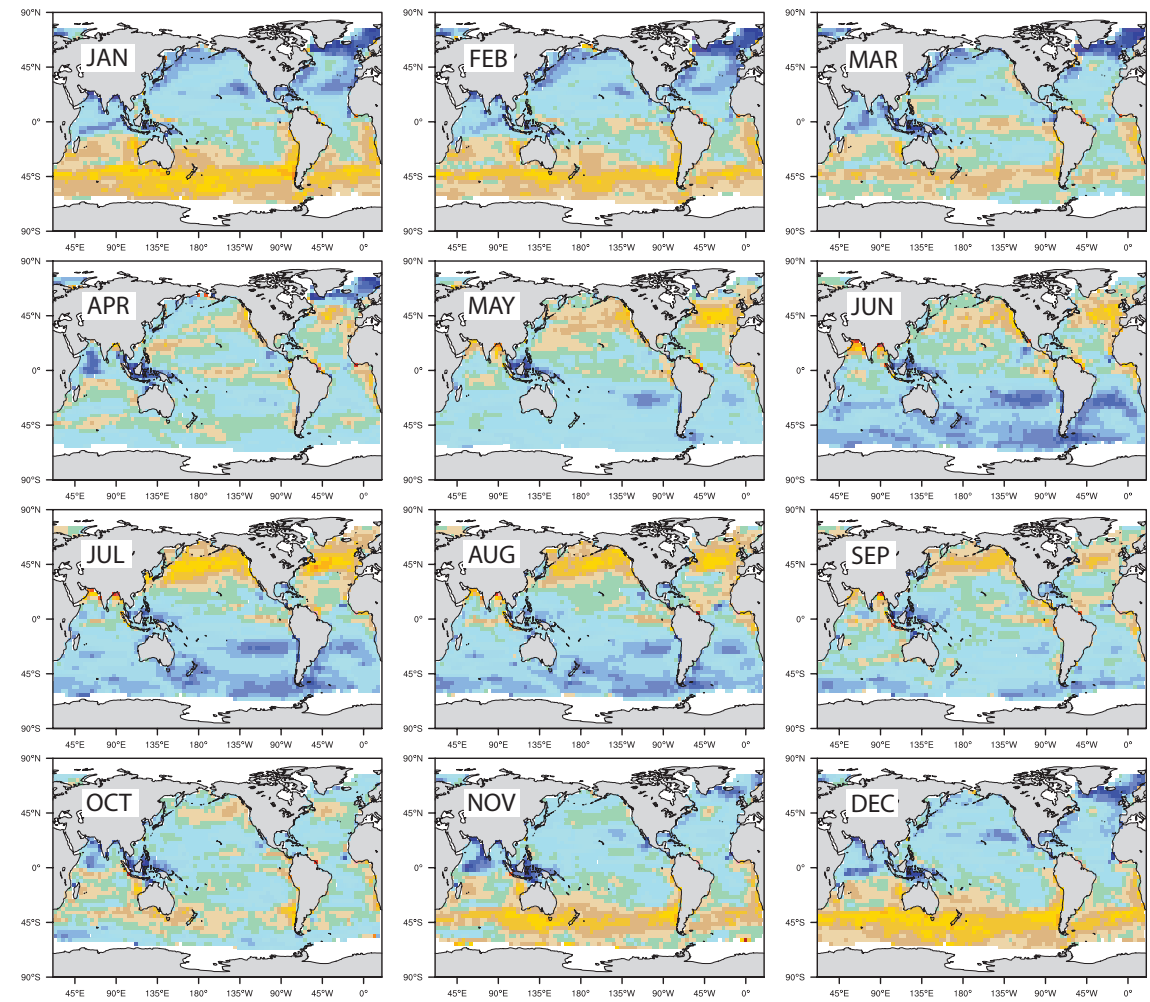

$\begin{array}{lllllllllll}1 & 1.08 & 1.16 & 1.24 & 1.32 & 1.4 & 1.48 & 1.56 & 1.64 & 1.72 & 1.8\end{array}$

Figure 1: The maps of the monthly mean enhancement factor from the Data Wave. 

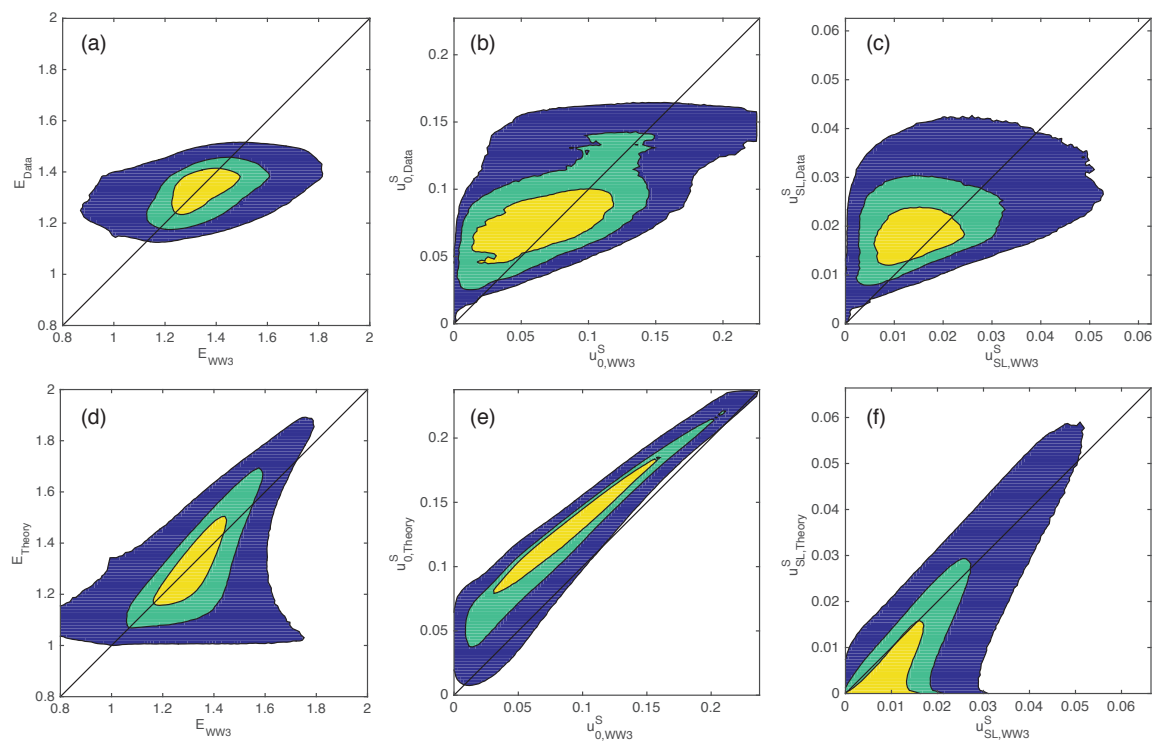

Figure 2: The density-shaded scatter plots of $(\mathrm{a}, \mathrm{d})$ the enhancement factor, $\mathcal{E}$, (b,e) surface Stokes drift magnitude $\left(\mathrm{m} \mathrm{s}^{-1}\right), u_{0}^{\mathrm{S}}$ and $(\mathrm{c}, \mathrm{f})$ surface layer averaged Stokes drift magnitude $\left(\mathrm{m} \mathrm{s}^{-1}\right), u_{\mathrm{SL}}^{\mathrm{S}}$. Comparisons are made between $(\mathrm{a}, \mathrm{b}, \mathrm{c})$ Data Wave and WW3, and (d,e,f) Theory Wave and WW3. The scatter plots are generated from the 6-hourly output data over the last 10 years of the simulation WW3. The values for Data Wave is obtained from the 10-year monthly average, followed by a linear interpolation to expand the data to 6hourly frequency. The values for Theory Wave is calculated from (25). For all the figures, yellow, green and blue represent the highest $0-30 \%, 31-60 \%$ and $61-90 \%$ centered distribution, respectively. 

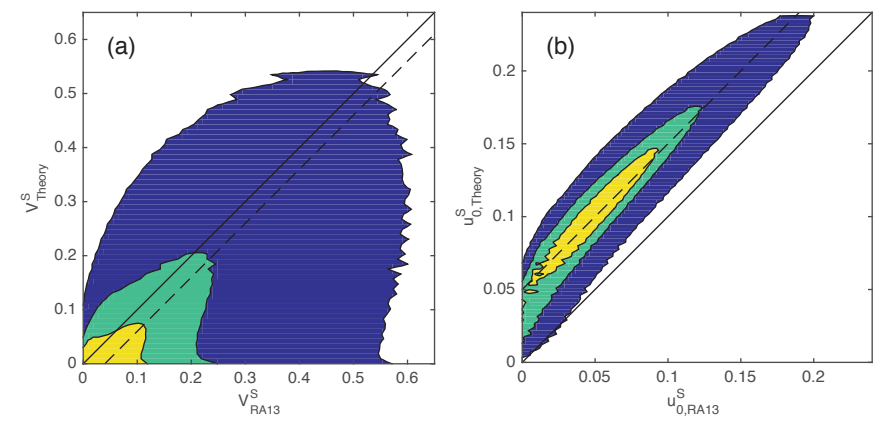

Figure 3: The density-shaded scatter plots of (a) total Stokes transport, $V^{\mathrm{S}}$ and (b) surface Stokes drift, $u_{0}^{\mathrm{S}}$, generated from one year of the 3-hourly output data from RA13. Comparison is made between the Theory Wave prediction and the model diagnosed data in RA13. The total Stokes transport and surface Stokes drift in Theory Wave are calculated from (19) and (23), respectively, with $c_{1}=0.667$ and $c_{2}=0.016$. See Fig. 2 for the explanation for the colors. 

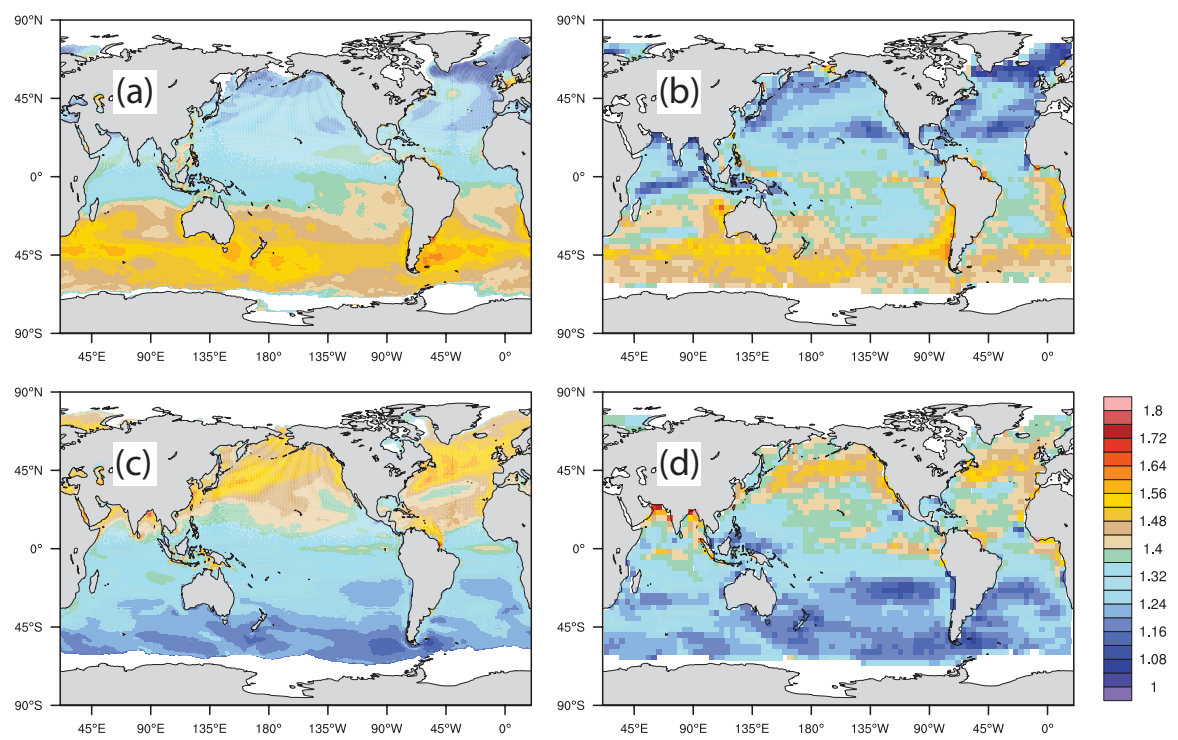

Figure 4: Comparison of the January $(a, b)$ and July $(c, d)$ mean enhancement factor map between Theory Wave $(a, c)$ and Data Wave $(b, d)$. 

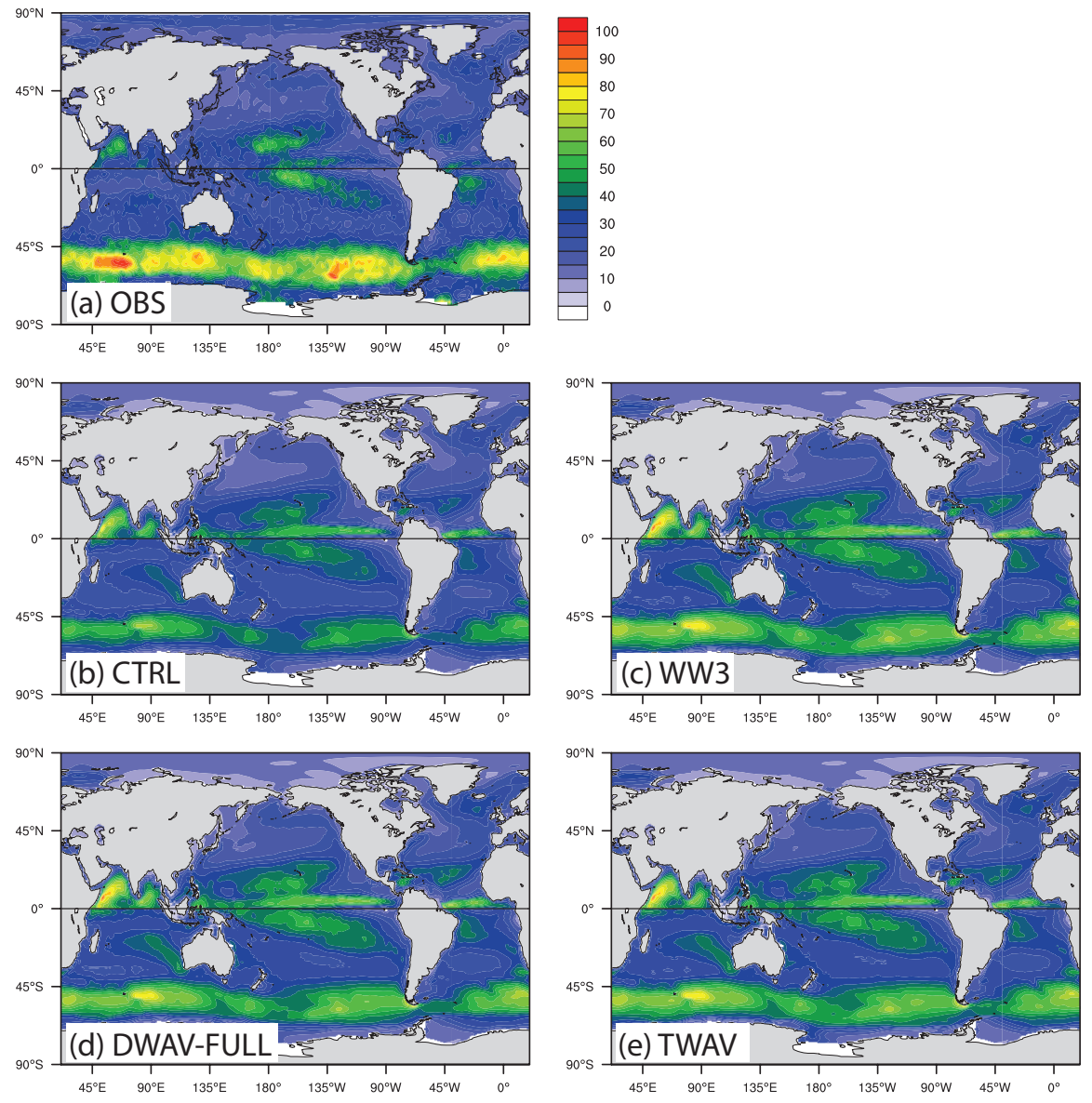

Figure 5: Impact of Langmuir mixing on the summer mean mixed layer depth (MLD; $\mathrm{m}$ ) for both hemispheres. Subfigure (a) shows the observation from de Boyer Montégut et al. (2004), updated to include the ARGO data to 2012. (b) shows the case CTRL, (c) the case WW3, (d) the case DWAV-FULL and (e) the case TWAV. MLDs are averaged over Jul., Aug. and Sep. for the Northern Hemisphere and Jan., Feb. and Mar. for the Southern Hemisphere. 

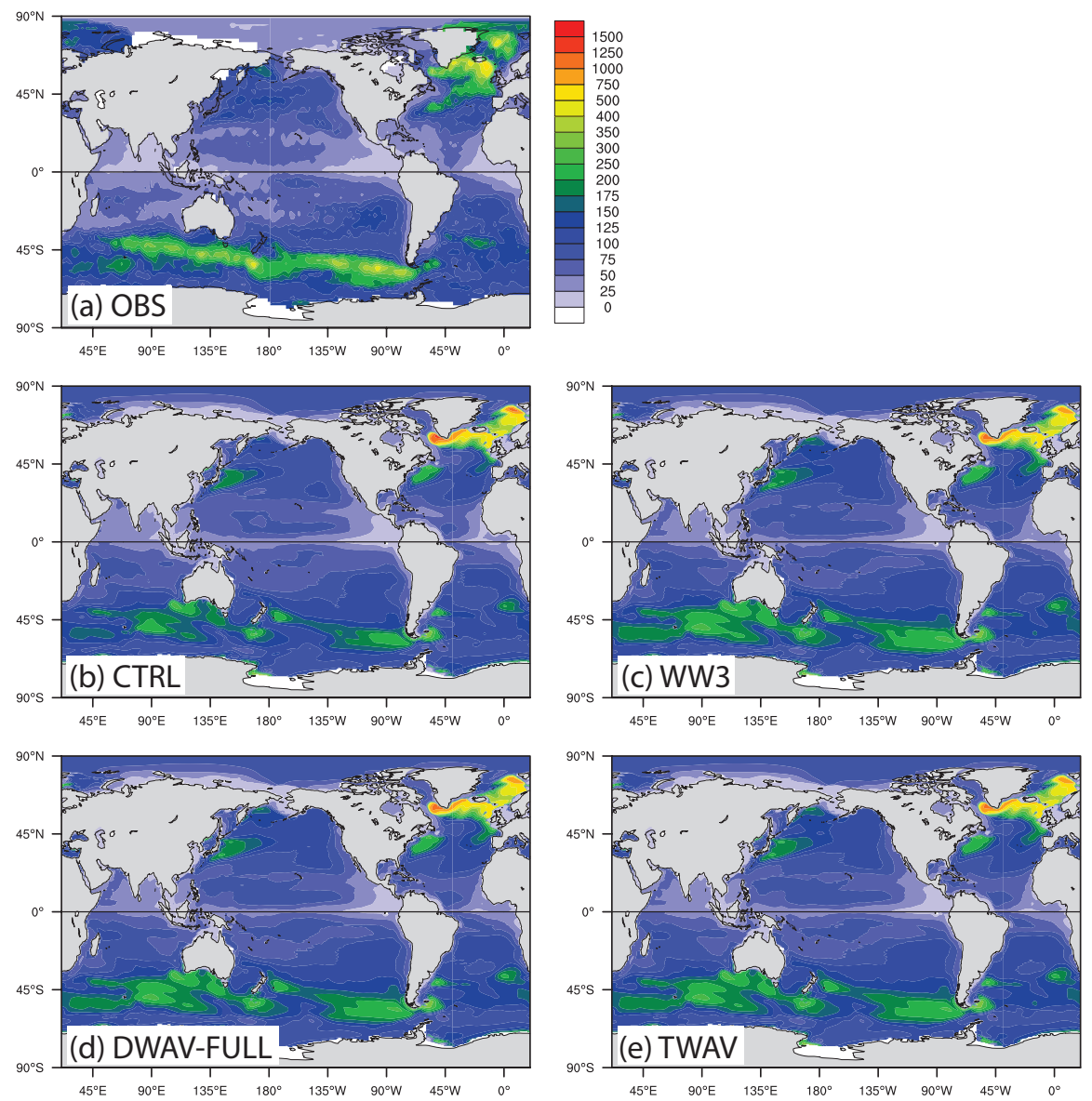

Figure 6: Same as Fig. 5, but for winter mean MLD. Note the different color scalings here. 

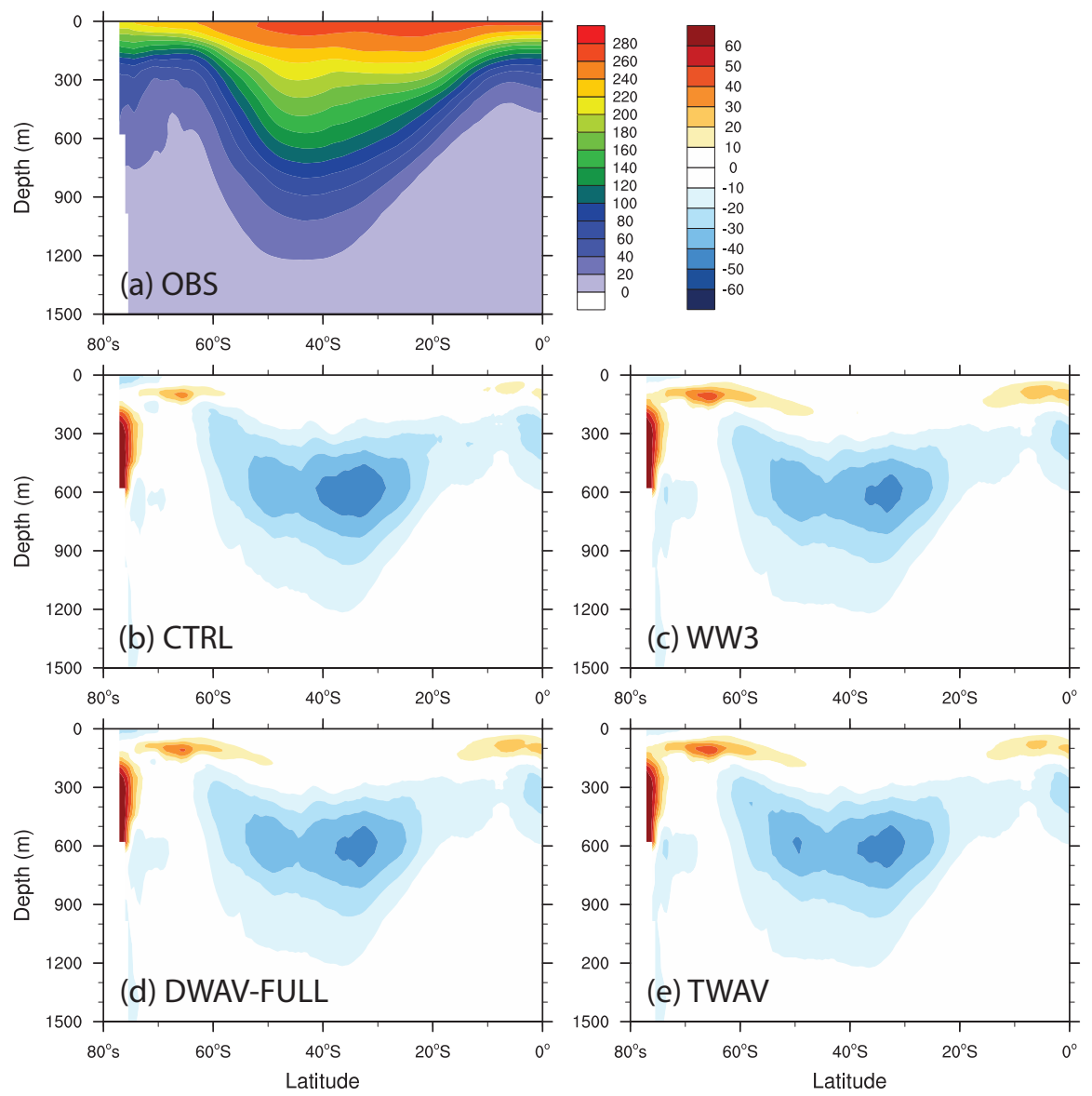

Figure 7: Impact of Langmuir mixing on the zonal mean pCFC-11 (patm) in the Southern Hemisphere. (a) shows the observation from Key et al. (2004). (b)-(e) show the anomaly from the observation for the cases CTRL, WW3, DWAV-FULL and TWAV, respectively. 

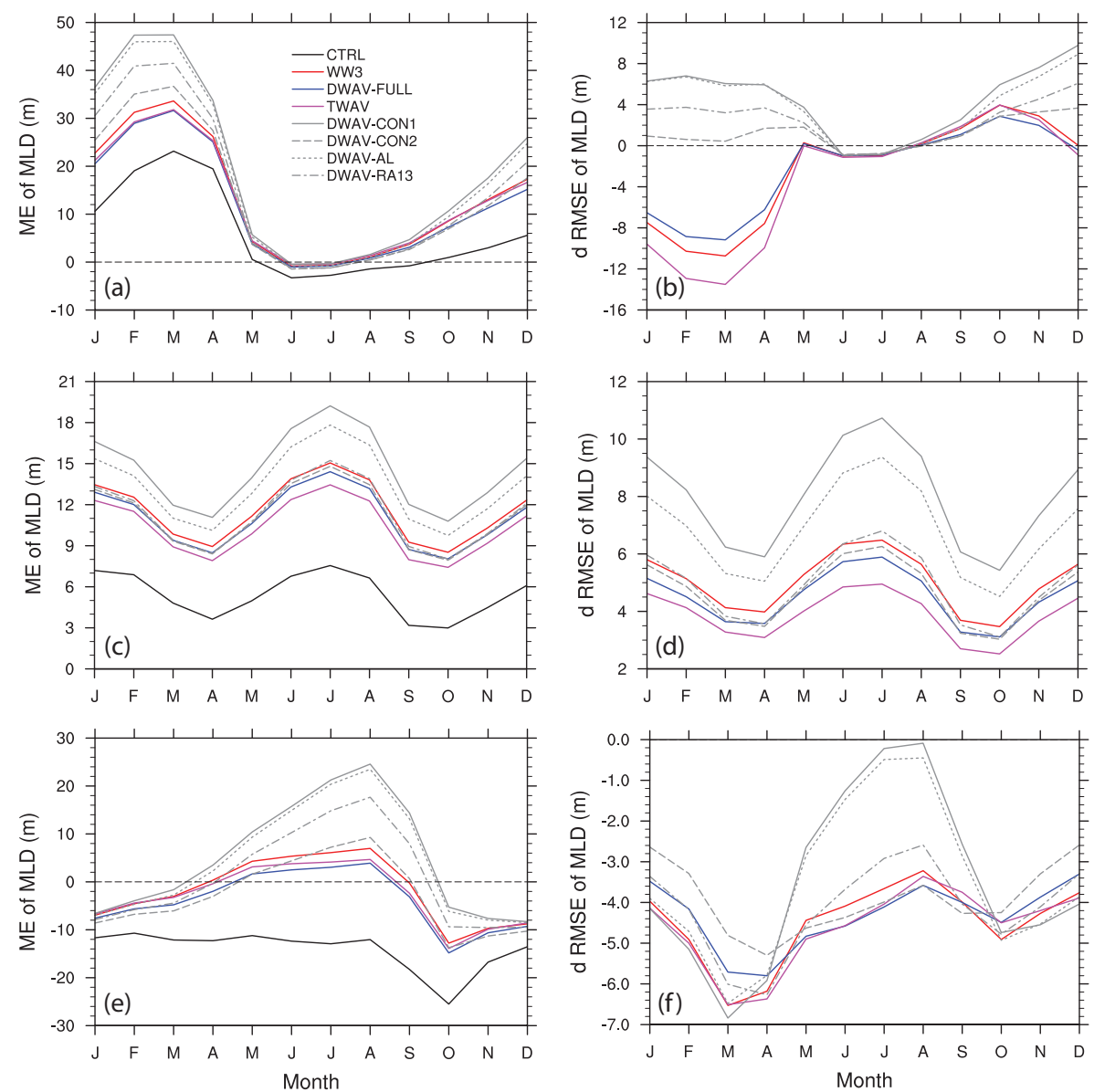

Figure 8: The seasonal cycle of the mean MLD biases versus observation (a, c, e) and the corresponding reductions in root mean square errors (RMSE) after including Langmuir mixing (b, d, f), averaged over $(\mathrm{a}, \mathrm{b}) 30^{\circ} \mathrm{N}-90^{\circ} \mathrm{N},(\mathrm{c}, \mathrm{d}) 30^{\circ} \mathrm{S}-30^{\circ} \mathrm{N}$ and (e, f) $90^{\circ} \mathrm{S}-30^{\circ} \mathrm{S}$. The control simulation (CTRL) without Langmuir mixing effect is colored in black, the simulation with WAVEWATCH III (WW3) in red, the Data Wave (DWAV-FULL) in blue and the Theory Wave (TWAV) in purple. Overlaid solid, dashed, dotted and dash-dotted curves in gray are for DWAV-CON1, DWAV-CON2, DWAV-AL and DWAV-RA13, respectively. Note that (b), (d) and (f) show the differences in RMSE as compared with CTRL. 

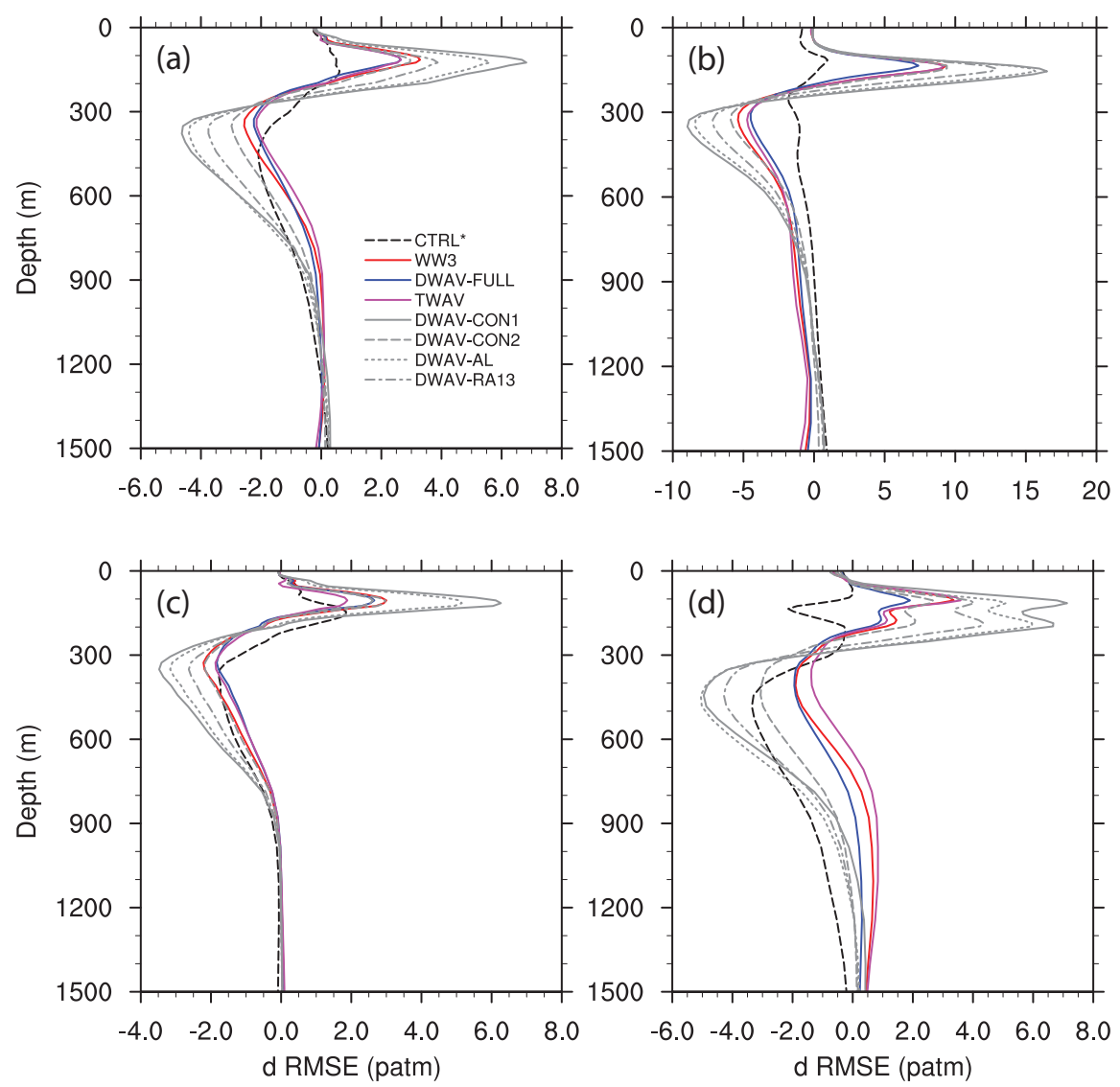

Figure 9: The vertical profiles of the RMSE reductions of pCFC-11 (patm) as compared with CTRL over regions: (a) Global; (b) $30^{\circ} \mathrm{N}-90^{\circ} \mathrm{N}$; (c) $30^{\circ} \mathrm{S}-30^{\circ} \mathrm{N}$; (d) $90^{\circ} \mathrm{S}-30^{\circ} \mathrm{S}$. The color coding is the same with Fig. 8, except an additional black dashed line here, which illustrates the difference of pCFC-11 RMSE in CTRL between the year 1995 and the year 1994 (see text for more details). All RMSEs are calculated against the observation from Key et al. (2004), which basically represents the year 1994 . 

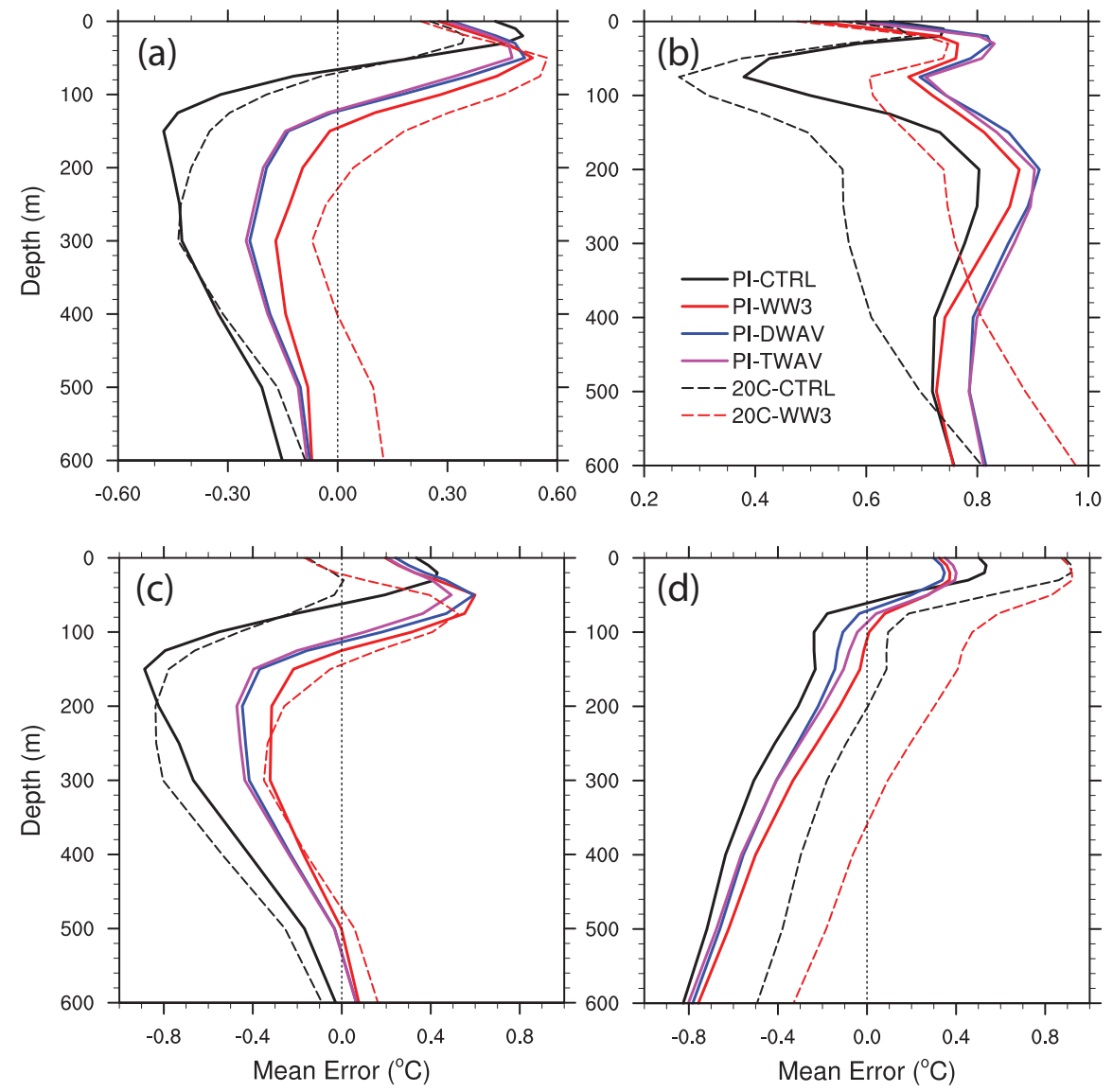

Figure 10: The vertical profiles of the mean error of temperature $\left({ }^{\circ} \mathrm{C}\right)$ as compared with the observation from Steele et al. (2001) (PHC3.0) over regions: (a) Global; (b) $30^{\circ} \mathrm{N}-90^{\circ} \mathrm{N}$; (c) $30^{\circ} \mathrm{S}-30^{\circ} \mathrm{N} ;\left(\right.$ d) $90^{\circ} \mathrm{S}-30^{\circ} \mathrm{S}$. 


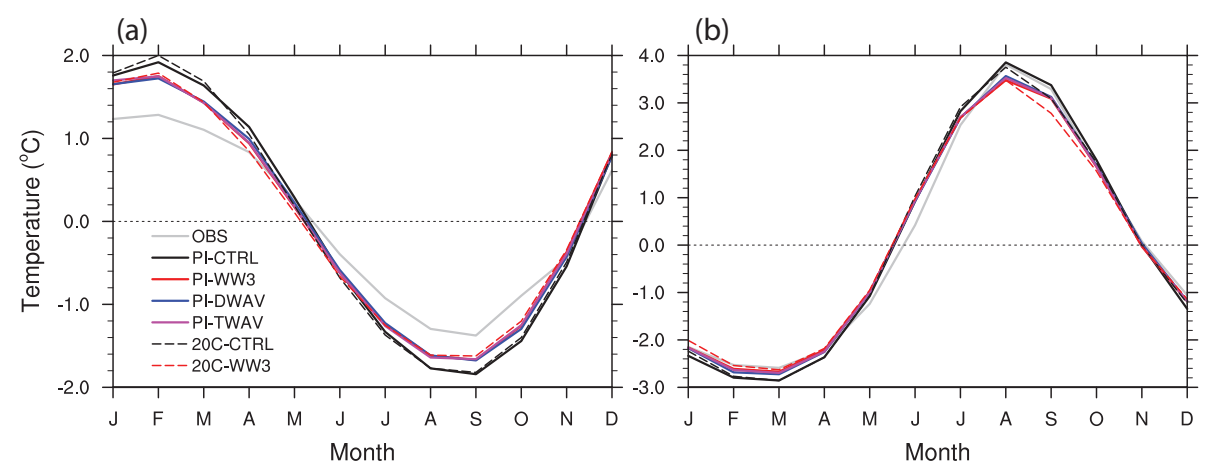

Figure 11: The seasonal cycle (annual mean subtracted) of the sea surface temperature (SST; ${ }^{\circ} \mathrm{C}$ ) averaged over (a) $90^{\circ} \mathrm{S}-30^{\circ} \mathrm{S}$ and (b) $30^{\circ} \mathrm{N}-90^{\circ} \mathrm{N}$. 